\title{
Algorithms for collision-free navigation of mobile robots in complex cluttered environments: a survey Michael Hoy $\dagger^{*}$, Alexey S. Matveev $\ddagger$ and Andrey V. Savkin $\dagger$
}

$\dagger$ School of Electrical Engineering and Telecommunication, University of New South Wales, Sydney, Australia

$\ddagger$ Department of Mathematics and Mechanics, Saint Petersburg University, St. Petersburg, Russia

(Accepted February 2, 2014. First published online: March 4, 2014)

\begin{abstract}
SUMMARY
We review a range of techniques related to navigation of unmanned vehicles through unknown environments with obstacles, especially those that rigorously ensure collision avoidance (given certain assumptions about the system). This topic continues to be an active area of research, and we highlight some directions in which available approaches may be improved. The paper discusses models of the sensors and vehicle kinematics, assumptions about the environment, and performance criteria. Methods applicable to stationary obstacles, moving obstacles and multiple vehicles scenarios are all reviewed. In preference to global approaches based on full knowledge of the environment, particular attention is given to reactive methods based on local sensory data, with a special focus on recently proposed navigation laws based on model predictive and sliding mode control.
\end{abstract}

KEYWORDS: Path planning; Navigation, Multi-robot systems; Motion planning; Mobile robots; Control of robotic systems.

\section{Introduction}

Navigation of unmanned vehicles is a classic research area in robotics, which gives rise to a whole variety of approaches well documented in the literature. Both single and multiple coordinated unmanned vehicles offer great perspectives in many applications, such as industrial, official, and agricultural automation; search and rescue; surveillance and inspection; for a more comprehensive list of potential applications, we refer the reader to [252, 279]. In all these cases, navigation involves a series of common problems, with collision avoidance in some form being almost universally needed. This review broadly documents methods relating to navigation of unmanned vehicles as applicable to collision avoidance, which still remains a focus of active research. Among various proposals, this paper surveys the recent ones and those capable of rigorous collision avoidance, i.e., for which it is rigorously proven that collisions never occur, provided that some technical assumptions are fulfilled.

Provable collision avoidance is a highly desirable trait in navigation systems since it shows that the system safely operates under a broad range of conditions, rather than just those considered during testing.

Overall, navigation systems underlaid by more general assumptions about the problem would be considered superior. Some examples of assumptions are listed as follows:

- Vehicle models vary in complexity from velocity-controlled linear models to realistic car-like models (see Section 2). For example, collision avoidance for velocity-controlled models is simpler since the vehicles can halt instantly if required; however this is physically unrealistic. This means a more complex model that better characterizes the vehicle is desirable for use during analysis.

\footnotetext{
* Corresponding author. E-mail: mch.hoy@gmail.com
} 
- Different levels of knowledge about the obstacles and other vehicles are required by different navigation strategies. This ranges from abstracted obstacle set information to the actual nature of realistic noisy sensor data obtained from range-finding sensors. In addition to the realism of the sensor model, the actual sensing requirements significantly vary between approaches-in some cases, methods that only utilize limited information (such as the minimum distance to the obstacle) are required.

- Different assumptions about the shape of static obstacles have been proposed. To ensure correct behavior when operating near an obstacle with limited information available, it is often necessary to presume smoothness of the obstacle boundary. This means that approaches with more flexible assumptions about obstacles would be more widely applicable to real-world scenarios.

- Uncertainty is always present in real robotic systems, and proving collision avoidance under an exact vehicle model will not always imply that the associated correct behavior will be exhibited when implemented. To reflect this, assumptions can be made describing bounded disturbance from the nominal model, bounded sensor errors, and the presence of communication errors. A review of the types of uncertainty present in vehicle systems is available, see, e.g., [57].

While being able to prove collision avoidance under broad circumstances is one of the most desirable features, examples of other navigation law features that determine their effectiveness are listed as follows:

- Navigation laws that provably satisfy the vehicle's goals are highly desirable. When possible, this can be generally shown by providing an upper bound on the time in which the vehicle will complete a finite task, however conservative this may be. However, proving goal satisfaction is possibly less critical than collision avoidance, so long as it can be experimentally demonstrated that non-convergence is virtually non-existent.

- In many applications, the computational ability of the vehicle is limited, and approaches with lower computational cost are favored. However, with ever increasing computer power, this concern is mainly focused toward small, fast vehicles such as miniature unmanned air vehicles (UAVs, which require fast update rates, challenging the limited computational faculties available). In practice, the computational burden is highly dependent on other factors such as programming efficiency, which impedes direct comparison of the computational performance of different approaches. However in general, it can be said that controllers based on closed-form expressions are faster than those based on local planning, which are faster than those based on global planning.

- Many navigation laws are constructed in continuous time. Virtually all digital control systems are updated in discrete time, thus navigation laws constructed in discrete time are more suitable for direct implementation.

The key distinction between different approaches is the amount of information they have available about the workspace. When full information is available about the obstacle set and a single vehicle is present, global path planning methods may be used to find the optimal path. When only local information is available, sensor-based methods are used. A subset of sensor-based methods is reactive methods, which may be expressed as a mapping between the sensor state and control, with no memory present.

While global methods are not directly applicable to the problem of collision avoidance in unknown environments, we have included discussion of many of these approaches in this review. This is because many of these methods could be adapted for unknown environments, sometimes with relatively little modification. A review of collision avoidance methods would be incomplete without including them.

Recently, model predictive control (MPC) architectures have been applied to collision avoidance problems, and this approach seems to show great potential in providing efficient navigation, and easily extends to robust and nonlinear problems (see Section 3 ). They have many favorable properties compared to the commonly used artificial potential field (APF) methods and velocity obstaclebased methods, which could be generally more conservative when extended to higher order vehicle models. MPC continues to be developed and demonstrates many desirable properties for sensor-based navigation, including applications to boundary following problems (see Section 4.1.2), avoidance of moving obstacles (see Section 5), and coordination of multiple vehicles (see Section 6). 
In this paper, we review both local and global approaches, together with approaches applicable to multiple vehicles and moving obstacles. We also explore in detail the various types of vehicle and sensor models, together with assumptions about obstacles and their movement.

\subsection{Exclusions}

Because of the breadth of this research, we will not review the following areas, only providing a brief summary where necessary:

- Mapping algorithms. Mapping is becoming very popular in real-world applications, where exploration of unknown environments is required (see, e.g., [71, 115, 272]). While they are extremely useful, and many collision avoidance techniques require some kind of map to operate, this review would be too broad if we were to include them - a vast number of approaches have been proposed in recent years. Additionally, this review is focused on local collision avoidance, and in many cases it would be unnecessary to build a map to achieve this. However, we will mention the DistBug class of algorithms (see, e.g., [87, 198]), which is possibly the simplest examples of non-reactive navigation (a single point is stored to detect circumnavigation of a particular obstacle).

- Path tracking systems. This continues to be an important, non-trivial problem in the face of realistic assumptions, and several types of collision avoidance approaches assume the presence of an accompanying path following navigation law. However, it is an auxiliary problem to collision avoidance, and the necessary material may be found in the collision avoidance literature, whenever path following is needed (see, e.g., [54, 205]).

- High-level decision making. The most common, classic approach to real-world implementations of unmanned vehicles seems to be a hierarchical structure where a high-level planner provides general directions, while a low-level navigation layer prevents collision and attempts to follows the commands given by the higher layer (in pure reactive schemes the high level is effectively replaced with some heuristic). While this type of decision making is required in some situations to show convergence, it becomes too abstracted from our basic goal of showing collision avoidance. We only wish to delegate convergence tasks if they can be achieved within the same basic navigation framework, see, e.g., [313].

- Motor schema, subsumption, and behavior-based architectures. In general, these attempt to replicate the behavior of animals, by having a number of different behaviors that can be selected as necessary according to the situation (see, e.g., $[10,11,162,175])$. While these have historic importance, they focus on applications more general than just collision avoidance (avoidance tends to be achieved via some type of APF method, which are covered separately in this review). Also, they do not tend to be associated with rigorously provable collision avoidance, though some approaches that achieve provable avoidance could be considered by means of evolved versions of these methods.

- Planning algorithm implementations. Many of the approaches discussed may be used with several types of planning algorithms, thus the discussion may be separated. This review effectively focuses on the parameters and constraints given to path planning systems, and the subsequent use of the output. Many other surveys have explored this topic, see, e.g., [97, 156].

- Specific tasks (including swarm robotics, formation control, target searching, area patrolling, and target visibility maintenance). In these cases, the primary objective is not proving collision avoidance between agents, and these approaches are only included in cases where the underlying collision avoidance approach is not documented elsewhere.

- Iterative learning, fuzzy logic, and neural networks. While these are all important areas and are well suited to some applications (see, e.g., [114]), and also generate promising experimental results, it is generally more difficult to obtain guarantees of motion safety when applied directly to vehicle motion, see, e.g., [98]. However note these may indirectly be used in the form of planning algorithms, which may be incorporated into the approaches discussed in this review.

The remainder of the survey is structured as follows. In Section 2, the problem of navigating cluttered environments is described. In Section 3, MPC-based navigation systems are described. In Section 4, methods of sensor-based navigation are introduced; in Section 5, methods of dealing with moving obstacles are reviewed. Section 6 deals with the case of multiple cooperating vehicles. Finally, Section 7 presents brief conclusions. 


\section{Problem Considerations}

In this section, we outline some of the real-world factors that influence the design of vehicle navigation systems. It would be considered an advantage for a particular navigation system to take some of these factors into account, since this makes it easier to translate into a real-world implementation.

\subsection{Environment}

Our definition of a cluttered environment consists of a two- or three-dimensional workspace, which contains a set of simple, closed, untransversable obstacles, which the vehicle is not allowed to overlap with. The area outside the obstacle is considered homogenous and equally easy to navigate. Examples of cluttered environments may include offices, man-made structures, and urban environments. An example of classification of objects in an urban environment is available. ${ }^{68}$

The vehicle is spatially modeled as either a point, circle, or polygon in virtually all approaches. Polygons can be conservatively bounded by a circle, so polygonal vehicle shapes are generally only used for tight maneuvering around closely packed obstacles, where an enclosing circle would exclude marginally viable trajectories.

\subsection{Vehicle kinematics}

There are many types of vehicles that must operate in cluttered environments, such as ground vehicles, UAVs, surface vessels, and underwater vehicles. Most vehicles can be generally categorized into three types of kinematic models—holonomic, unicycle, and bicycle - where the differences are characterized by different kinematic constraints. Reviews of various vehicle models are available, see, e.g., [100, 101, 129, 188]. We use the term dynamic to describe models based on the resolution of physical forces, while kinematic describes models based on more abstracted control inputs.

- Holonomic kinematics. These describe vehicles that have control capability in any direction. Holonomic kinematics are encountered on helicopters, and certain types of wheeled robots equipped with omni-directional wheels. Holonomic motion models have no notion of body orientation for the purposes of path planning, and only the Cartesian coordinates are considered. However, orientation may become a consideration when applying the resulting navigation law to real vehicles, though this is decoupled from planning.

- Unicycle kinematics. These describe vehicles that are associated with a particular angular orientation, which determines the direction of the velocity vector. Changes to the orientation are limited by a turning rate constraint. Unicycle models can be used to describe various types of vehicles, such as differential drive wheeled mobile robots and fixed wing aircraft, see, e.g., [166, 167].

- Bicycle kinematics. These describe a car-like vehicle, which has a steerable front wheel separated from a fixed rear wheel. Kinematically this implies that the maximum turning rate is proportional to the vehicles speed. This places an absolute bound on the curvature of any path the vehicle may follow regardless of speed. This constraint necessitates high-order planning to navigate in confined environments.

It should be noted that nonholonomic constraints are generally only a limiting factor at low speed; for example, realistic vehicles would likely be also subjected to absolute acceleration bounds, which limit maneuverability at high speed. More complex kinematics are also possible, but uncommon.

In addition to these basic variants of kinematics, the associated linear and angular variables may be either velocity-controlled or acceleration-bounded. Vehicles with acceleration-bounded control inputs are in general much harder to navigate; velocity-controlled vehicles may stop instantly at any time if required.

When predicting a vehicle's actual motion, these nominal models are invariably subject to disturbance. The type of disturbance that may be modeled depends on the kinematic model:

- Holonomic models. Disturbance models commonly consist of bounded additions to the translational control inputs, see, e.g., [223].

- Unicycle models. Bounded addends to the control inputs can be combined with a bounded difference between the vehicle's orientation and actual velocity vector, see, e.g., [141]. More realistic models of differential drive mobile robots are also available, which are based on modeling wheel slip rates (see, e.g., [5, 14]). 
- Bicycle models. Disturbance can be modeled as slide slip angles on the front and rear wheels (see, e.g., [176]). Alternatively, more realistic disturbance models of car-like vehicles are available, which include factors such as suspension and type adhesion (see, e.g., [28, 303]).

Vehicles with bicycle kinematics or vehicles with minimum speed constraints will be subject to absolute bounds on their path curvature. This places some global limit on the types of environments they can successfully navigate through, see, e.g., [25, 29]. When lower bounds on allowable speed are present, the planning system is further complicated. For example, instead of halting, the vehicle must follow some holding pattern at the termination of a trajectory.

\subsection{Sensor data}

Most autonomous vehicles must base their navigation decisions on data reported by on-board sensors, which provide some information about the vehicles' immediate environment. The main types of sensor model are listed as follows:

- Abstract sensor models. This label is applied to any method where the navigation law is assumed to know, with certainty, whether a given point lies within a given obstacle set. Usually any occluded regions (without a line of sight to the vehicle) are considered to be part of the obstacle. Though it is impossible to fully identify the shape of an obstacle using a physical sensor, currently some Light Detection and Ranging Device (LiDAR) sensors have accuracy high enough for any sampling effects to be of minor concern (e.g., simple piecewise linear interpolation of the detected points would approximate the obstacle quite well). However, when lower resolutions are present, this model may be unsuitable for navigation law design.

- Ray-based sensor models. These models inform the navigation law of the distance to the obstacle in a finite number of directions around the vehicle, see, e.g., [143, 193, 275]. This is a more physically realistic model of laser-based sensors compared to the abstract sensor model, and may be suitable for determining the effect of low-resolution sensors. A reduced version of this model is used in some boundary following applications, where only a single detection ray in a fixed direction (relative to the vehicle) is present.

- Minimum distance measurements. This sensing model reports the distance to the nearest obstacle point. This may be realized by certain types of wide-aperture acoustic or optic flow sensors. Using this type of measurement necessarily leads to less efficient movement patterns during obstacle avoidance, i.e., it is not immediately clear which side of the vehicle the obstacle is on (see, e.g., [181]).

- Tangent sensors. This sensor model reports the angles to visible edges of an obstacle as seen by the vehicle (see, e.g., [246, 265]). This can be realized from a camera sensor, provided a method of detecting obstacle edges from a video stream is available (see, e.g., [113]).

- Optic flow sensors. This model reports the average rate of pixel flow across a camera sensor (see, e.g., [33, 104]). From this rate of pixel flow, a navigation command may be expressed, and good practical results may be achieved. While these types of sensors are very compact, unfortunately rigorously provable collision avoidance may not be possible.

There is a large number of ways in which noise and distortion may be compensated for in these models, and these tend to be quite specific to individual approaches. Some examples include linear quadratic Gaussian, ${ }^{286}$ sliding-mode control-based tracking, ${ }^{110}$ and H-infinity tracking. ${ }^{206}$

\subsection{Optimality criteria}

There are several different methods of preferentially choosing one possible trajectory over another. Many path optimization algorithms may be implemented with various such measures or combinations of measures. Common possibilities are listed below:

- Minimum distance. This is used in the majority of path planning schemes as it can be decoupled from the achievable velocity profile of the vehicle. For moving between two configurations without obstacles, the classic result of Dubins describes the optimal motion of curvature-bounded vehicles. ${ }^{70}$ In this case, the optimal path consists of a sequence of no more than three maximal turns or straight segments. Other similar results are available for vehicles with actuated speed, ${ }^{217}$ and for velocity-controlled, omni-directional vehicles. ${ }^{15}$ However, these results are of little direct use in path planning, since obstacles have a complex effect on any optimal path. It was shown in 
ref. [153] for the case when acceleration constraints are absent, and in ref. [237] for the general case, that the minimum distance path may be constructed from the Tangent Graph of an obstacle set.

- Minimum wheel rotation. This applies to differential drive wheeled mobile robots, and in most cases is only subtlety different from the minimum distance formulation. However, it may perform better in some situations, especially when fine movements are required (see, e.g., [49]).

- Minimum time. Calculating the transversal time of a path depends on the velocity profile of the vehicle, and thus includes kinematic (and possibly dynamic) constraints. In most situations, it would be more appropriate than minimum distance for selecting the trajectories that complete tasks in the most efficient manner. It is often used in MPC-based approaches, see, e.g., [223].

- Minimum control effort. This may be more suitable for vehicles operating in limited energy environments, e.g., spacecraft or passive vehicles, however it is invariably combined with another measure for non-zero movement.

- Optimal surveillance rate. In unknown environments, it may be better to select trajectories that minimize the occluded part of the environment (see, e.g., [276]). In cases where occluded parts of the environment must be treated as unknown dynamic obstacles, this could allow a more efficient transversal, though it would unavoidably rely on stochastic inferences about the unknown portion of the workspace. This may be an interesting area of future research.

Other examples of requirements that can be applied to trajectories include higher order curvature rate limits, which may be useful to produce smoother trajectories (see, e.g., [12]).

\subsection{Biological inspiration}

Researchers in the area of robot navigation in complex environments find much inspiration from biology, where the problem of controlled animal motion is a central one. This is prudent since biological systems are highly efficient and refined, while the equivalent robotic systems are in relative infancy. Animals, such as insects, birds, or mammals, are believed to use simple, local motion control rules that result in remarkable and complex intelligent behaviors. Therefore, biologically inspired or biomimetic algorithms of collision-free navigation play an important part in this research field.

In particular, the idea of the navigation along an equiangular spiral and the ideas of local obstacle avoidance strategies proposed in refs. [240, 270, 271]) are also inspired by biological examples. It has been observed that peregrine falcons, which are among the fastest birds on the earth, plummet toward their targets at speeds of up to 200 miles an hour along an equiangular spiral. ${ }^{277}$ Furthermore, in biology, a similar obstacle avoidance strategy is called "negotiating obstacles with constant curvatures" (see, e.g., [148]). An example of such a movement is a squirrel running around a tree. These ideas in reactive collision avoidance robotic systems are further discussed in Section 4.1. Furthermore, the sliding-mode control-based methods of obstacle avoidance discussed in Section 4.1 are also inspired by biological examples such as the near-wall behavior of a cockroach. ${ }^{39}$ Another example is the Bug family algorithms that are also inspired by bugs' behavior on crawling along a wall; see Section 4.1.3. Motor Schema and subsumption architectures may be considered to be biologically inspired, however, these are not covered in this review (see, e.g., [10, 11, 162, 175]).

Optical flow navigation is another important class of biologically inspired navigation methods. The remarkable ability of honeybees and other insects like them to navigate effectively using very little information is a source of inspiration for control strategies' design. In particular, the work of Srinivasan et al. ${ }^{260}$ explains the use of optical flow in honeybee navigation, where a honeybee makes a smooth landing on a surface without the knowledge of its vertical height above the surface. As it is commonly observed in insects' flights, the navigation command is derived from the average rate of pixel flow across a camera sensor (see, e.g., $[33,104]$ ).

Other approaches that use a camera to locate targets may also be classed as biologically inspired. For example, the navigation approach in ref. [157] allows a robot to converge to a target solely using measurements of the target's location in the camera frame.

Many ideas in multi-robot navigation are also inspired by biology, where the problem of animal aggregation is central in both ecological and evolutionary theory. Animal aggregations, such as schools of fish, flocks of birds, groups of bees, or swarms of social bacteria, are believed to use simple, local motion coordination rules at the individual level that result in remarkable and complex intelligent behavior at the group level (see, e.g., $[32,80]$ ). Such intelligent behavior is expected from 


\begin{tabular}{|c|c|c|c|c|c|}
\hline & & Single vehicle & Moving obstacles & Multi-vehicle & Boundary following \\
\hline \multirow[t]{3}{*}{ MPC } & Standard & $U^{*}, A, M, F, T$ & $\mathrm{U}^{*}, \mathrm{~A}, \mathrm{M}, \mathrm{F}, \mathrm{T}^{*}$ & & \\
\hline & Robust & $\mathrm{U}^{*}, \mathrm{~A}, \mathrm{M}, \mathrm{F}, \mathrm{R}, \mathrm{T}$ & & & \\
\hline & Local planning & $U, A, M, F, R$ & $A, M, F$ & & $U, A, M, F, T$ \\
\hline \multirow[t]{2}{*}{ DMPC } & Distributed optimisation & & & $U^{*}, A, M, R$ & \\
\hline & Synchronous & & & $U^{*}, A, M, F, R$ & \\
\hline \multirow[t]{3}{*}{ Boundary following } & Minimal information & & $\mathrm{U}^{*}, \mathrm{~A}^{*}, \mathrm{M}, \mathrm{F}, \mathrm{T}^{*}$ & & $\mathrm{U}, \mathrm{A}^{*}, \mathrm{M}, \mathrm{F}, \mathrm{T}$ \\
\hline & Full information & & $\mathrm{U}^{*}, \mathrm{~A}^{*}, \mathrm{M}, \mathrm{F}, \mathrm{T}^{*}$ & & $U, A^{*}, M, F, T$ \\
\hline & Bug algorithms & & & & $\mathrm{U}, \mathrm{F}, \mathrm{T}$ \\
\hline \multirow[t]{2}{*}{ Velocity obstacle } & $\mathrm{VO} / \mathrm{NLVO}$ & $\mathrm{U}^{*}, \mathrm{~A}^{*}, \mathrm{~F}, \mathrm{~T}^{*}$ & $\mathrm{U}^{*}, \mathrm{~A}^{*}, \mathrm{M}, \mathrm{F}, \mathrm{T}^{*}$ & & \\
\hline & DRCA/RCA & & $U, A^{*}, M^{*}, F, R^{*}$ & $U, A^{*}, M, F, R, T^{*}$ & \\
\hline \multirow[t]{4}{*}{ Other } & APF & $U, A^{*}, M, F, T^{*}$ & $U, A^{*}, M, F$ & $A^{*}, M^{*}, F, T$ & $U, M, F, T$ \\
\hline & Tangent following & $U, A^{*}, M, F, T$ & $\mathrm{U}^{*}, \mathrm{~A}^{*}, \mathrm{M}, \mathrm{F}, \mathrm{T}$ & & $\mathrm{U}, \mathrm{A}^{*}, \mathrm{M}, \mathrm{F}, \mathrm{T}$ \\
\hline & Other reactive & $U, A^{*}, M, F, T$ & $\mathrm{U}, \mathrm{A}^{*}, \mathrm{M}, \mathrm{F}, \mathrm{T}^{*}$ & $\mathrm{U}, \mathrm{F}$ & $\mathrm{U}, \mathrm{M}, \mathrm{F}, \mathrm{T}$ \\
\hline & Hybrid logic & $A, M, F, T$ & & $\mathrm{~A}, \mathrm{M}, \mathrm{F}, \mathrm{T}$ & \\
\hline
\end{tabular}

\begin{tabular}{|ll|}
\hline Key: & \\
$U$ & Unknown environment \\
A & Acceleration bounded \\
M & Unicycle or bicycle model \\
$F$ & Fast computation \\
R & Robust to disturbance \\
T & Provably convergent \\
$*$ & Some disadvantages \\
\hline
\end{tabular}

Fig. 1. Summary of the traits characterizing the methods discussed in this review. This table makes some subjective, generalizing judgments, and should not be considered a definitive comparison. The symbol * means the method has some limitations and would not be completely characterized as exhibiting the corresponding trait (and thus further work could possibly be done in these areas).

very large scale robotic systems. Because of decreasing costs of robots, interest in very large scale robotic systems is growing rapidly. In such systems, robots should exhibit some forms of cooperative behavior. We discuss it further in Section 6.

There is also some evidence that approaches resembling MPC are used by higher animals to avoid obstacles. ${ }^{4}$ It seems natural to achieve collision avoidance using some type of planning into the future, and MPC-based navigation laws are discussed in Section 3.

\subsection{Implementation examples}

There are many reviews of current applications and implementations of real-world vehicles, see, e.g., [123]. Because of length considerations we do not give an exhaustive list of reported applications, however we highlight one particular application.

Semi-autonomous wheelchairs and intelligent robotic hospital beds are a recent application in which a navigation law must be designed to prevent collisions while taking high-level direction inputs from the user, see, e.g., [34, 292, 294]. In this case, a fundamental concern for these intelligent wheelchairs is maintaining safety, thus the methods described in this review are highly relevant. Several original collision avoidance approaches were originally proposed for wheelchair applications, see, e.g., [294] (these are also discussed in Section 5.3.2).

\subsection{Summary of methods reviewed}

A very broad summary of the methods considered in this review is listed in Fig. 1, where the availability of certain traits is shown. This table makes some subjective, generalizing judgments, and should not be considered a definitive comparison. These methods will be discussed in much more detail in the remainder of this review.

\section{Model Predictive Control}

It should be said that if an obstacle-avoiding trajectory is planned off-line, there are many examples of path following systems that are able to robustly follow it, even if subjected to a bounded disturbance. However, the lack of flexibility means the environment would have to be perfectly known in advance, which is not conducive to on-line collision avoidance. 


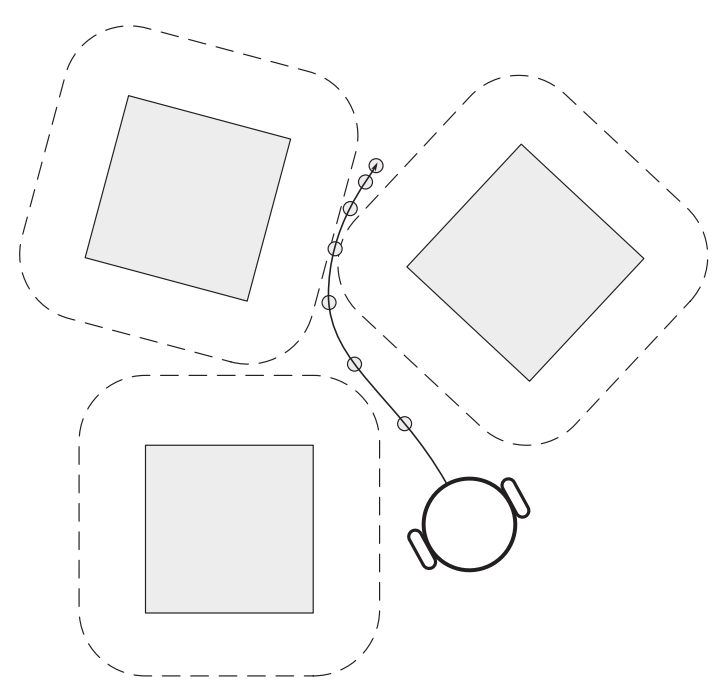

Fig. 2. Diagram of the MPC approach to vehicle navigation. At each control update, a path is planned into the future, and the initial control input corresponding to this trajectory is implemented on the vehicle.

MPC $^{1}$ is increasingly being applied to vehicle navigation problems. It is useful as it combines path planning with on-line stability and convergence guarantees, see, e.g., [187, 224]. This is basically done by performing the path planning process at every time instant, then applying the initial control related to the chosen trajectory to the vehicle (this process is illustrated in Fig. 2). In most cases, a partial path that terminates with an invariant vehicle state (the vehicle is stationary or in a loiter circle) is employed, which minimizes some cost-to-go function corresponding to the target. By repeating this process at every time step, the controller can be shown to be stable.

Note that in this section we mainly discuss approaches that are applicable to known environments. Many of these methods can be extended to cases where information is restricted; however discussion of MPC approaches applicable to unknown environments is reserved until Section 4.2.

\subsection{Robust MPC}

The key advantage of MPC lies with its robust variants, which are able to account for set bounded disturbance (and are most useful for vehicle navigation). These can be categorized into three main categories:

- Min-max MPC. In this formulation, the optimization is performed with respect to all possible evolutions of the disturbance, see, e.g., [243]. While it is the optimal solution to linear robust control problems, its high computational cost generally precludes it from being used for vehicle navigation.

- Constraint tightening MPC. Here, the state constraints are dilated by a given margin so that a trajectory can guaranteed to be found, even when disturbance causes the state to evolve toward the constraints imposed by obstacles (see, e.g., [136, 222, 223]). The basic argument shows existence of a future viable trajectory by using a feedback term, though a feedback input is not directly used for updating the trajectory. This is commonly used for vehicle navigation problems-for example, a system has been described where an obstacle-avoiding trajectory is found based on a minimization of a cost functional compromising the control effort and maneuver time. ${ }^{223}$ In this case, convergence to the target and the ability to overcome bounded disturbances can be shown.

- Tube MPC. This uses an independent reference model of the system, and employs a feedback system to ensure that the actual state converges to the nominal state (see, e.g., [140]). In contrast, the constraint tightening system would essentially take the nominal state to be the actual state at each time step. Tube MPC is more conservative than constraint tightening, since it would not take advantage of favorable disturbance. Thus, it does not offer significant benefits for vehicle navigation problems when a linear model is used. However, it is useful for robust nonlinear

${ }^{1}$ Equivalent to Receding Horizon Control (RHC). 
MPC (see, e.g., [186]), and problems where only partial state information is available (see, e.g., [243]). Also, any approach that includes path following with bounded deviation (see, e.g., [60]) is somewhat equivalent to tube MPC.

For robust MPC, the amount of separation required from the state constraints on an infinite horizon is determined by the robustly positively invariant (RPI) set, which is the set of all possible state deviations that may be introduced by disturbance while a particular disturbance rejection law is operating. Techniques have been developed to efficiently calculate the smallest possible RPI set (the minimal RPI set). ${ }^{215}$

If disturbance is Gaussian rather than set bounded, the MPC problem may be reformulated stochastically so the overall risk of collision is bounded to an arbitrary level, see, e.g., [30, 69].

\subsection{Nonlinear $M P C$}

The current approaches to MPC-based vehicle navigation generally rely on linear kinematic models, usually with double integrator dynamics. While many path planning approaches exist for vehicles with nonholonomic kinematics, it is generally harder to show stability and robustness properties. ${ }^{164}$ Approaches to robust nonlinear MPC are generally of the tube MPC type. ${ }^{186}$ In these cases, a nonlinear trajectory tracking system can be used to ensure that the actual state converges to the nominal state. A proposition has been made to also use sliding-mode control laws for the axillary system (for which disturbance rejection is typically easier to show), though such systems typically require continuous time analysis, see, e.g., [228].

In terms of vehicle navigation problems, examples of MPC that apply unicycle kinematics while having disturbance present have been proposed, see, e.g., [59, 60, 110, 311]. Similar methods guarantee convergence using passivity-based arguments. ${ }^{267}$ However, it seems that more general applications of nonlinear MPC to vehicle navigation problems should be possible.

There are other methods in which MPC may be applied to vehicle navigation problems other than performing rigorously safe path planning. In some cases, the focus is shifted toward controlling vehicle dynamics, see, e.g., [99, 208, 303]. These use a realistic vehicle model during planning, and are able to give good practical results, though guarantees of safety are currently easier with more kinematic models. In other cases, MPC may be used to regulate the distance to obstacles, see, e.g., [248] (however, this discussion is reserved until Section 4.1.4).

\subsection{Planning algorithms}

Global path planning is a relatively well-studied research area, and many thorough reviews are available, see, e.g., [97, 156]. MPC may be implemented with a number of different path planning algorithms. The main relevant measure of algorithm quality is completeness, which indicates whether calculation of a valid path can be guaranteed whenever one exists. Some common global path planning algorithms are summarized as follows:

- Rapidly exploring random trees. Creates a tree of possible actions to connect initial and goal configurations (see, e.g., [64, 121]). Some variants are provably asymptotically optimal. ${ }^{121}$

- Graph search algorithms. Examples include A* (see, e.g., [233]), D* (see, e.g., [128]), and fast marching (see, e.g., [89]). Most methods hybridize the environment into either a square graph, an irregular graph, ${ }^{117}$ or a Voronoi diagram ${ }^{89}$ (the latter is the skeleton of points, which separates all obstacles). A search can then be performed to calculate the optimal sequence of node transitions. In addition, this may be used as the first step to find a bounded area within which further path planning operations can take place. ${ }^{288}$

- Optimization of predefined paths. Examples include Bezier curves, ${ }^{256}$ splines, ${ }^{146}$ and polynomial basis functions. ${ }^{213}$ While these are inherently smoother, showing completeness may be more difficult in some situations.

- Artificial potential field methods. These methods are introduced in Section 4.3.1, as they are also ideally suited to on-line reactive navigation of vehicles (without path planning). These can also be used as path planning approaches, essentially by using more information about the environment (see, e.g., $[90,295])$. However, the resultant trajectories would not be optimal in general. 
- Mathematical programming and optimization. This usually is achieved using mixed integer linear programming (MILP) constraints to model obstacles as multiple convex polygons. ${ }^{2}$ Currently, this is commonly used for MPC approaches.

- Tangent graph-based planning. This limits the set of trajectories to cotangents between obstacles and obstacle boundary segments, from which the minimum distance path being found in general. ${ }^{237,274}$ The problem of shortest path planning in a known environment for unicycle-like vehicles with a hard constraint on the robot's angular speed was solved in ref. [237]. It is assumed that the environment consists a number of possibly non-convex obstacles with a constraint on the curvatures of their boundaries curvature and a steady target that should be reached by the robot. It has been proved that the shortest (minimal in length) path consists of edges of the so-called tangent graph. Therefore, the problem of the shortest path planning is reduced to a finite search problem.

- Evolutionary algorithms, simulated annealing, particle swarm optimization. These are based on a population of possible trajectories, which follow some update rules until the optimal path is reached (see, e.g., [27, 309]). However, these approaches seem to be suited to complex constraints, and may have slower convergence for normal path planning problems.

- Partially observable Markov decision processes. This calculates a type of decision tree for different realizations of uncertainty, and uses probabilistic sampling to generate plans that may be used for navigation over long time frames (see, e.g., [134]). However, this may not be necessary for all MPC-based navigation problems.

\section{Sensor-Based Techniques}

Sensor-based navigation techniques typically employ only limited local knowledge about the environment, exemplified by data obtained from range sensors, video cameras, or optic flow sensors. Global sensor-based planners use the sensory information (possibly combined with a priory knowledge, if applicable) to build a comprehensive model of the observed chunk of the environment and to find the best complete trajectory through it. ${ }^{21,145,289}$ Within this framework, several techniques (surveyed in, e.g., [133, 144]) have been developed even for dynamic scenes, including nonholonomic planners, velocity obstacles, ${ }^{79,144}$ and state-time space ${ }^{84,218}$ approaches. While the computational capabilities of unmanned vehicles are continually increasing, global planning problems will always feature NP-hardness (this mathematical seal for intractability was established for even the simplest problems of dynamic motion planning ${ }^{40}$ ). This is an important consideration especially for, e.g., micro UAVs, which must react quickly to new information despite having limited computational faculties available.

On the other hand, local path planners use on-board sensors to locally observe a nearest fraction of an unknown environment for iterative re-computation of a short-horizon trajectory. ${ }^{61,270}$ This reduces the calculation time and creates a potential for employment in certain real-time guidance systems. Many of the related techniques, such as the dynamic window, ${ }^{83,244}$ the curvature velocity, ${ }^{253}$ and the lane curvature ${ }^{196}$ approaches treat the obstacles as static. On the other side, approaches like velocity obstacles, ${ }^{79}$ collision cones,${ }^{44}$ or inevitable collision states ${ }^{85,207}$ assume a deterministic knowledge about the obstacle velocity and a moderate rate of its change.

In the marginal case where the planning horizon collapses into an infinitesimally short-time interval, local planner acts as a reactive feedback controller: it maps the current observation into the current control. Examples can be found where a general technique for design of reactive controllers is offered (see, e.g., [58]). Other examples include artificial potential approach, combined with slidingmode control for gradient climbing, ${ }^{77,152}$ and kinematic control based on polar coordinates and Lyapunov-like analysis. ${ }^{51} \mathrm{Up}$ to now, fully actuated, velocity-controlled robots were mostly studied in this area, the obstacles were interpreted as rigid bodies of the simplest shapes (e.g., disks ${ }^{51,77}$ or polygons ${ }^{152,170}$ ), the sensory data were assumed to be enough to determine the location of obstacle characteristic points concerned with its global geometry (e.g., the disk center ${ }^{51,77}$ or angularly most distant polygon vertex ${ }^{170}$ ) and to provide access to its full velocity. ${ }^{51,77,170}$ Furthermore, rigorous justification of the global convergence of the proposed algorithm is rarely encountered.

In this section, we mainly focus on local sensor-based planners, with particular attention given to reactive algorithms. 


\subsection{Obstacle avoidance via boundary following}

Temporarily following the boundary of an obstacle is a standard method employed by many obstacle avoidance algorithms. According to it, the robot directly pursues the main control objective until a threat of collision with an obstacle is detected. After this, the robot bypasses the obstacle by following its boundary with temporarily putting aside the main objective. The converse switch from boundary following holds as soon as a leaving condition is satisfied. This condition should guarantee that first, resuming direct pursuit of the objective does not cause a collision threat at least initially and second, the control objective will be ultimately achieved. In this context, the distance to the followed boundary is relatively insignificant; it may be time varying but should not excessively both decay and increase to ensure safety and to exclude collisions with companion obstacles, respectively. It should be also noted that boundary following is a self-contained navigation task of immediate interest for border patrolling and structure inspection, ${ }^{96,229}$ bottom following by autonomous underwater vehicles,${ }^{38}$ lane following by autonomous road vehicles, ${ }^{150}$ and other missions, where traveling along the boundary at a pre-specified distance from it is an essential requirement.

4.1.1. Distance-based. In many approaches, boundary following can be rigorously achieved by only measuring the minimum distance to the obstacle, see, e.g., [178, 181]. For example, in ref. [181], the navigation strategy was based on a sliding-mode navigation law; uses the minimum obstacle distance as input; and is suitable for guiding nonholonomic vehicles traveling at constant speed. In ref. [178], a similar sliding-mode argument is used, however the only required input is the rate of change of distance to the obstacle.

Other approaches have been proposed, which use a single obstacle distance measurement at a specific angle relative to the vehicle, see, e.g., [177, 273]. In refs. [270, 271], the navigation law calculation is based purely on the length of the detection ray, while in refs. [177, 273] additional information is also collected to estimate the tangential angle of the obstacle at the intersection point, while ${ }^{125}$ also requires the boundary curvature. Additional information would presumably result in improved behavior, however comparisons of closed loop performance are difficult.

Some other work using similar assumptions is focused on following straight walls, see, e.g., [24, $41,112,302]$. However, it seems that, at least theoretically, navigation laws capable of tracking contours are more general and therefore superior.

In most of these examples, the desired behavior can be rigorously shown. However, the common limitation is that the vehicle must travel at a constant speed, and that this speed must be set conservatively according to the smallest feature of the obstacle. In some cases, simple heuristics can partially solve this problem; by instructing the vehicle to instantly stop and turn in place if the obstacle distance becomes too small, collision may be averted. ${ }^{273}$

4.1.2. Sliding mode control. Due to the well-known benefits, such as stability under large disturbances, robustness against system uncertainties, good dynamic response, simple implementation, etc. ${ }^{280}$ the sliding-mode approach attracts an increasing interest in the area of mobile robotics. Examples include but are not limited to target following, ${ }^{183,239}$ environmental extremum seeking, ${ }^{182}$ and trajectory tracking. ${ }^{259,299}$

Sliding-mode-based boundary following with a pre-specified margin was addressed in refs. [177, 181] for a planar under-actuated nonholonomic vehicle or wheeled mobile robot modeled as unicycle. It travels with a constant speed $v$ and is controlled by the angular velocity $u$ limited by a given constant $\bar{u}$. The kinematics of the considered vehicles are classically described by the following equations:

$$
\begin{array}{ll}
\dot{x}=v \cos \theta & x(0)=x_{0} \\
\dot{y}=v \sin \theta & y(0)=y_{0} . \\
\dot{\theta}=u \in[-\bar{u}, \bar{u}] & \theta(0)=\theta_{0}
\end{array}
$$

Here, $x, y$ are the Cartesian coordinates of the vehicle in the world frame, $\theta$ gives its orientation, and the maximal angular velocity $\bar{u}$ is given. The model (1) is applied to many mechanical systems such as wheeled robots, aerial vehicles, missiles, etc.; see, e.g., [82, 157], and references therein. It captures the capability of the vehicle to travel forward with the given speed along planar curves whose curvature radius exceeds a certain threshold, which equals to $R:=v / \bar{u}$ in the case at hand. 
The workspace of the robot contains a domain $D \subset \mathbb{R}^{2}$. The distance from the vehicle current position $\boldsymbol{r}(t)=\operatorname{col}[x(t), y(t)]$ to $D$ is given by $d(t):=\operatorname{dist}_{D}[\boldsymbol{r}(t)]$, where

$$
\operatorname{dist}_{D}(\boldsymbol{r}):=\min _{\boldsymbol{r}^{\prime} \in D}\left\|\boldsymbol{r}-\boldsymbol{r}^{\prime}\right\|, \quad\left\|\boldsymbol{r}-\boldsymbol{r}^{\prime}\right\|:=\sqrt{\left(x-x^{\prime}\right)^{2}+\left(y-y^{\prime}\right)^{2}}
$$

for $\boldsymbol{r}=\operatorname{col}(x, y), \boldsymbol{r}^{\prime}=\operatorname{col}\left(x^{\prime}, y^{\prime}\right)$, the symbol $\|\cdot\|$ stands for the standard Euclidean vector norm, and min is achieved if $D$ is closed, which is assumed.

The boundary following problem is as follows. Find a control law that asymptotically drives the vehicle to the required distance $d(t) \rightarrow d_{0}>0$ to the domain $D$ and ensures the vehicle traveling along the domain boundary $\partial D$ at the given speed $v$. During the entire maneuver, the distance from the vehicle to the domain should constantly exceed $d(t) \geq d_{\text {safe }}$ the given safety margin $d_{\text {safe }} \in\left[0, d_{0}\right)$.

In ref. [181], solution to this problem was given in the form of the following discontinuous controller, which originates from the equiangular navigation and guidance law: ${ }^{271}$

$$
\begin{aligned}
& u(t)=\bar{u} \cdot \operatorname{sign}\left\{\dot{d}(t)+\chi\left[d(t)-d_{0}\right]\right\}, \quad \text { where } \\
& \chi(z):=\left\{\begin{array}{ll}
\gamma z & \text { if }|z| \leq \delta \\
v_{*} \operatorname{sign}(z) & \text { if }|z|>\delta
\end{array} \quad\left(v_{*}:=\gamma \delta\right)\right.
\end{aligned}
$$

is the linear function with saturation, $\operatorname{sign}(\alpha):=1$ for $\alpha>0, \operatorname{sign}(0):=0$, and $\operatorname{sign}(\alpha):=-1$ for $\alpha<0$, whereas $\gamma>0$ and $\delta>0$ are controller parameters. This control law assumes that the vehicle has access to the current distance $d(t)$ to $D$ and the rate $\dot{d}(t)$ at which this measurement evolves over time (computed via, e.g., numerical differentiation), but no further sensing capabilities are needed.

The paper ${ }^{181}$ discloses requirements to the boundary $\partial D$ necessary for the vehicle with a limited turning radius to be capable of tracking the $d_{0}$-equidistant curve at the given speed. It is proved that whenever these minimal requirements are met in a slightly enhanced form and are extended on the transient, the controller (3), (4) does ensure global convergence to and subsequent following along the required equidistant curve, with always respecting the safety margin $d_{\text {safe }}$ during the transient. This holds for properly tuned controller parameters, with explicit recommendations on their choice being provided. For such parameters, the vehicle driven by this controller first undergoes circular motion with a maximal steering actuation $u= \pm \bar{u}$. This motion necessarily ends with commencement of sliding motion over the surface $\dot{d}(t)+\chi\left[d(t)-d_{0}\right]=0$ in the state space $\{(x, y, \theta)\}$ of (1), which will never terminate and ensure the desired convergence $d(t) \rightarrow d_{0}$ as $t \rightarrow \infty$. Proper saturation injected by the function $\chi(\cdot)$ plays an important role here since the relation $\dot{d}(t)+\chi\left[d(t)-d_{0}\right]=0$ is unrealistic if $\chi(\cdot)$ exceeds the maximal feasible value $v$ of the rate $\dot{d}$.

The control law (3), (4) is fed by input variables whose computation may require wide-aperture sensing and intensive pre-processing, like many other controllers proposed in the extensive literature on reactive path and boundary following. For example, reactive (i.e., mapless) vision navigation, which excellent surveys are available in refs. [33,63], employs the capability of the visual sensor to capture and memorize a whole chunk of the environment and is typically based on extraction of certain image features and estimation of their motion within a sequence of images, which requires intensive image processing. Some other examples of perceptually and computationally demanding input variables not confined to the area of visual navigation include the closest point on the obstacle boundary, the distance to this point, or the value of another function determined by the entire boundary; see, e.g., [24, 41, 75, 112, 165, 174, 178, 188, 230, 306, 307, 312] for representative samples. Another such variable employed by many proposed controllers, see, e.g., $[125,165,307]$, is the boundary curvature, which is particularly sensitive to corruption by measurement noises since this is a second derivative property.

Sliding-mode controllers fed by non-demanding input variables immediately provided by certain perceptually deficient sensory systems are offered in refs. [177, 184]. The paper ${ }^{177}$ deals with the problem of boundary following with a pre-specified margin by the vehicle (1) based on only the distance $d_{\perp}$ along and the reflection angle $\varphi$ of the ray perpendicular to the vehicle centerline. Such situation holds if, e.g., the measurements are supplied by several range sensors rigidly mounted on the vehicle body at nearly right angles from its centerline or by a single sensor scanning a nearly perpendicular narrow sector. This perception scheme is used in some applications to reduce the 
complexity, cost, weight, and energy consumption of the sensor system and to minimize detrimental effects of mechanical external disturbances on the measurements. However, the related deficit of sensor data makes most of known navigation solutions inapplicable in this case especially if this deficit is enhanced by a low computation power of the digital processor, which is characteristic for, e.g., miniature robots. Moreover, this gives rise to special challenges, like inability to detect a threat of head-on collision in certain situations (e.g., motion over a circle centered at the obstacle boundary) or strong sensitivity of the overall output of the sensor system to the vehicle posture.

In ref. [177], solution to the boundary following problem was given in the form of the following switching control law:

$$
u=\left\{\begin{array}{l}
-\bar{u} \text { if } S:=\varphi+\chi\left[d_{\perp}-d_{0}\right] \leq 0 \\
u_{+}:=\min \left\{\bar{u} ; v d_{\perp}^{-1}\right\} \text { if } S>0
\end{array},\right.
$$

where $\chi(\cdot)$ is still a linear function with saturation (4). The first line in (5) dictates the vehicle to make a clockwise turn with the maximal steering angle. In accordance with the second line, the vehicle moves counter-clockwise over the circle centered at the boundary, thus keeping the distance $d_{\perp}$ and the reflection point constant, if this distance exceeds the minimal turning radius $R=v / \bar{u}$. Otherwise, a counter-clockwise turn with the maximal steering actuation is carried out. It is shown that under some technical and partly unavoidable assumptions, the control law (5) does ensure the desired non-local convergence $d(t) \rightarrow d_{0}$ as $t \rightarrow \infty$ with respecting the safety margin $d_{\text {safe. }}$. In doing so, sliding-mode effects still play an essential role though the overall trajectory may be more complicated than the union of an initial turn and subsequent sliding motion, like in the case from [181].

Some other reactive controllers fed by data from perpendicularly mounted sensors have been proposed in refs. [125, 270]. The control law from [270] is aimed at pure obstacle avoidance, with no objective to follow the boundary with a pre-specified margin. Boundary following with a given margin by a Dubins-car-like vehicle (1) was addressed in ref. [125] via a hybrid (non-sliding-mode) strategy of switching between Lyapunov-based highly nonlinear control laws in order to overcome singularities caused by concavities of the tracked curve. In doing so, restrictions on the steering control were neglected by assuming that the vehicle is capable of making arbitrarily sharp turns, and noise-sensitive estimates of the boundary curvature were essentially employed.

Another situation concerned with perceptually deficient sensory systems may occur in the cases where the obstacle is constituted by an area that the vehicle is forbidden rather than incapable to penetrate. This may be radioactively or chemically contaminated area, the region of hazardous weather conditions like hurricanes or that filled by fire, vapor, poisonous gases, or contaminant clouds. Some such areas can be sensed as a whole by means of, e.g., remote capabilities of satellites or radars, which opens the door to computation or estimation of the distance to the obstacle, the curvature of its boundary, or other variables used as regular inputs by many available controllers. However, there are many scenarios where such observation is troublesome, e.g., because of obstructions in urban, forest, or indoor environments, or is not precise or frequent enough, like, e.g., in fire spreads, ${ }^{42}$ or is infeasible since observation is physically based on an immediate contact with the sensed entity, like a transparent fluid, gas, or radiation. For many of such cases, the "forbidden" area is that where the value of a unknown scalar environmental field $F(\boldsymbol{r}) \in \mathbb{R}, \boldsymbol{r} \in \mathbb{R}^{2}$ exceeds a given threshold $f_{0}$, and the sensors observe this value in a point-wise fashion, i.e., at the location of the sensor. In this case, boundary following takes the form of tracking an environmental level set (isoline) $I\left(f_{0}\right):=\left\{\boldsymbol{r}: F(\boldsymbol{r})=f_{0}\right\}$, and the control objective shapes into $f(t):=F[\boldsymbol{r}(t)] \stackrel{t \rightarrow \infty}{\longrightarrow} f_{0}$. This includes pure boundary following based on the distance (2) to a given obstacle $D$ as a particular case: $F(\boldsymbol{r})=-\boldsymbol{d i s t}_{D}(\boldsymbol{r}), f_{0}=-d_{0}$.

The problem of tracking environmental level sets has gained much interest in the control literature. Most of the related publications fall into two categories: ${ }^{116}$ one of them assumes an access to the field gradient or derivative-dependent ${ }^{2}$ information (see, e.g., $[154,169,261,308]$ ) and the other is the gradient-free approach (see, e.g., [9, 16, 26, 42]). Gradient-based contour estimation by multiple sensor platforms was studied in refs. [122, 169, 261]. The centralized methods developed in refs. $[122,169]$ originate from the "snake" algorithms in image segmentation; the cooperative algorithm from [261] takes care for optimal spread of the sensors over the estimated contour to minimize

${ }^{2}$ For example, the curvature of the isoline. 
latency. Artificial potential approach based on direct access to the gradient was applied in ref. [154] to decentralized cooperative boundary tracking by a team of velocity-controlled points. By assuming the ability to move over the isoline for granted, as well as access to its curvature and tangent, [266] proposes an algorithm for uniform cooperative distribution of mobile sensors along the estimated boundary in order to ensure its optimal polygonal approximation. Collaborative estimation of the gradient and Hessian of a scalar field corrupted by noise was used in ref. [308] to develop a control law driving the center of a rigid formation of multiple mobile sensors along level curves.

In practical setting, derivative information is in fact unavailable in many cases, whereas its estimation requires access to the field values at several locations. Teams of mobile sensors have extended capacity of the latter thanks to collaborative sensing and data exchange. However, even in this case, limitations on communication may require a mobile sensor to operate individually for considerable time and distance. The practical scenario of a single mobile sensor with access to only point-wise measurements of only the field value is the main motivation for gradient-free approaches.

Gradient-free bang-bang-type steering controllers were reported in refs. [116, 310]. The control is via switches between alternative steering angles depending on whether the current field value is above or below the threshold of interest. In ref. [16], similar in spirit approach with a larger set of alternatives was applied to an underwater vehicle equipped with a profile sonar. These methods typically result in a zigzagging behavior. This is coherent with the approach to the gradient climbing that arranges for acquisition of extra information via extra maneuvers by "dithering" the sensor position. 37,53,305 However, systematic superfluous maneuvers may be required for this, whereas the multiple sensor scenario means more complicated and costly hardware. A method to control an unmanned aerial vehicle based on segmentation of the infrared local images of the forest fire was proposed in ref. [43]. These works rely, more or less, on heuristics and in fact offer no rigorous and completed justification of the proposed control laws. Linear powered device (PD) controller fed by the current field value was proposed in ref. [17] for steering a unicycle-like vehicle along a level curve of a field given by a radial harmonic function, and a local convergence result was established for a vehicle with unlimited control range.

Sliding-mode approach was employed in ref. [184] to design a controller that ensures tracking the desired environmental level set, does not employ gradient estimation, and is non-demanding with respect to both computation and motion. It is assumed that the on-board control system of the vehicle (1) has access only to the field value $f(t):=F[x(t), y(t)]$ at the vehicle current location and is capable to access the rate $\dot{f}(t)$ at which this measurement evolves over time $t$. The following analog of the control law (3) was proposed:

$$
u(t)=\operatorname{sign}\left\{\dot{f}(t)+\chi\left[f(t)-f_{0}\right]\right\} \bar{u} .
$$

The paper ${ }^{184}$ discloses requirements to the isoline $I\left(f_{0}\right)$ necessary for the vehicle $(1)$ with limited turning capacity to be capable of tracking the isoline. It is rigorously proved that whenever these minimal requirements are met in a slightly enhanced form and are extended on the transient, the controller (6) does ensure global convergence to and subsequent following along the required isoline provided that the controller parameters are properly tuned, with explicit recommendations on their choice being offered. For such parameters, the vehicle driven by this controller first undergoes circular motion with a maximal steering actuation $u= \pm \bar{u}$ and then proceeds with sliding motion over the surface $\dot{f}(t)+\chi\left[f(t)-f_{0}\right]=0$ in the state space, which will never terminate and ultimately ensure the desired convergence $f(t) \rightarrow f_{0}$ as $t \rightarrow \infty$.

The major obstacle to implementation of sliding-mode controllers like (3), (5), and (6) is a harmful phenomenon called "chattering," 280 i.e., undesirable finite frequency oscillations around the ideal trajectory due to un-modeled system dynamics and constraints. The problem of chattering elimination and reduction has an extensive literature (see, e.g., [149] for a survey). It offers a variety of effective approaches, including continuous approximation of the discontinuity, inserting low-pass filters/observers into the control loop, combining sliding-mode and adaptive control techniques, higher order sliding modes, etc. The issue of chattering was addressed in refs. [177, 181, 184] via computer simulations and real-world experiments with a Pioneer P3-DX mobile robot $^{3}$. Under continuous

\footnotetext{
${ }^{3}$ http://www.mobilerobots.com/researchrobots/pioneerp3dx.aspx
} 
approximation of the signum in (3), (5), (6) by a linear function with saturation, the authors reported no chattering.

Up to this point, the focus of the current subsection was on boundary following itself. The completed reactive control strategy of target reaching with obstacles' avoidance that employs boundary following as a hint to bypass en-route obstacles can be found in ref. [181]. The considered scenario assumes that the workspace of the vehicle (1) contains a steady point target $\boldsymbol{T}$ and several disjoint obstacles $D_{1}, \ldots, D_{k}$. The objective is to drive the vehicle to the target through the obstacle-free part of the plane $\mathbb{R}^{2} \backslash\left\{D_{1} \cup \cdots \cup D_{k}\right\}$. The proposed strategy consists in switching between the boundary following law (3), with $d(t)$ replaced by $d_{i}(t):=\operatorname{dist}_{D_{i}}[\boldsymbol{r}(t)]$ for a properly chosen $i$, and straight moves to the target:

$$
u(t)=0 .
$$

The rule for switching between (7) and (3) employs two given parameters ${ }^{4} \epsilon>0$ and $C>d_{0}+\epsilon$, where $C$ is the distance to an obstacle at which boundary following is commenced; its termination is allowed only if the vehicle is close enough to the obstacle: $d_{i} \leq d_{0}+\epsilon$. Specifically, the rule for switching between (7) and (3) is as follows:

R1 Switching (7) $\mapsto(3)$ (with $d(t):=d_{i}(t)$ ) occurs when the distance from the vehicle to the obstacle $D_{i}$ reduces to $C$.

R2 Switching (3) (with $\left.d(t):=d_{i}(t)\right) \mapsto$ (7) occurs when $d_{i}(t) \leq d_{0}+\epsilon$ and the vehicle is headed for the target.

To exclude the situation where the rule $\mathbf{R 1}$ becomes active simultaneously for several obstacles, the parameter $C$ should be less than half the minimal spacing between the obstacles. Furthermore, this rule tacitly assumes that the vehicle has access to the target bearing.

Under some technical assumptions, it is shown that the proposed control strategy does bring the vehicle to the target in a finite time with always respecting the safety margin $d_{i}(t) \geq d_{\text {safe }} \forall t, i$. The proofs are basically indifferent to the particulars of the law (3) so that the convergence has a solid potential to remain true if (3) is replaced by another convergent boundary following algorithm respecting the same margins during transients. The aforementioned assumptions stipulate in particular that the obstacles are convex and substantially spaced, and any obstacle $D_{i}$ that obstructs the view at the target from some point of another obstacle $D_{j}$ is totally closer to the target than $D_{j}$.

Sliding-mode boundary following was in effect concerned in ref. [178], though the proposed control strategy does not explicitly offer to follow the obstacle boundary. The paper ${ }^{178}$ addresses reactive navigation of the nonholonomic under-actuated robot (1) to a steady point-wise target through an arbitrarily shaped maze-like environment. The vehicle always has access to the relative bearing $\beta$ of the target ${ }^{5}$ and the distance $d$ to the nearest point of the maze whenever $d$ does not exceed a given sensing range $d_{\text {range }}$, but no further sensing capabilities are assumed. The proposed control law is constituted of the following bio-inspired reflex-level rules:

(s.1) At considerable distances from the obstacle,

(a) turn toward the target as fast as possible;

(b) move toward the target when headed for it;

(s.2) In close proximity of the obstacle,

(c) Follow (a,b) when moving away from the obstacle;

(d) Otherwise, quickly avert the collision threat by making a sharp turn.

Studies of target pursuit in animals, ranging from dragonflies to humans, have suggested that they often use pure pursuit method (s.1) to catch both steady and moving targets. The obstacle avoidance rule (s.2) is also inspired by biological examples such as the near-wall behavior of a cockroach. ${ }^{39}$

\footnotetext{
${ }^{4} \epsilon$ is tunable, but is typically chosen to be small.

5 The angle from the vehicle centerline to the target line of sight.
} 
Mathematically, the examined strategy is described by

$$
u=\bar{u} \times\left\{\begin{array}{l|l}
\operatorname{sign} \beta & \text { if } d>d_{\uparrow}(\text { mode } \mathfrak{A}) \\
\left\{\begin{array}{ll}
\operatorname{sign} \beta & \text { if } \dot{d}>0 \\
-\sigma & \text { if } \dot{d} \leq 0
\end{array} \mid \text { if } d \leq d_{\uparrow}(\operatorname{mode} \mathfrak{B})\right.
\end{array} .\right.
$$

Here, $\sigma$ and $d_{\uparrow} \in\left(0, d_{\text {range }}\right)$ are controller parameters, $\sigma$ can assume the values " +1 " or " -1 " and gives the turn direction in (d), $d_{\uparrow}$ regulates mode switching: $\mathfrak{A} \mapsto \mathfrak{B}$ when $d$ reduces to $d_{\uparrow} ; \mathfrak{B} \mapsto \mathfrak{A}$ when $d$ increases to $d_{\uparrow}$. When mode $\mathfrak{B}$ is switched on, $\dot{d} \leq 0$; if $\dot{d}=0$, the "turn" submode $u:=-\sigma \bar{u}$ is set up. In the basic version of the algorithm, $\sigma= \pm 1$ is fixed.

It is shown in ref. [178] that the rules (s.1) and (s.2) constitute a basically effective strategy: they do bring the vehicle through a simply connected arbitrarily shaped maze to the target. This property will be discussed in detail in the next subsection. Now we limit ourselves to a remark that though these rules do not explicitly recommend to follow the maze boundary, temporarily boundary following results from the interplay between (c) and (d) in the sliding-mode fashion in certain circumstances. Specifically, it is shown that the obstacle avoidance maneuver (i.e., motion within uninterrupted mode $\mathfrak{B}$ ) consists of finitely many motions along equidistant curves of the maze boundary interspersed by moves toward the target in straight lines. Switches between boundary following and straight moves to the target, as well as the distances to the boundary for "equidistant" parts are "automatically," though implicitly, set up by the rules (s.1) and (s.2). Though these distances may vary over the chain of these parts, the vehicle does follow the boundary in a certain direction (determined by $\sigma$ ) during the entire mode.

4.1.3. Bug algorithms. The Bug-family algorithms ${ }^{118-120,126,139,147,159,160,163,200,201,231,232}$ originated in refs. $[158,161]$ are among the first target reaching methods for which global convergence in complex scenes has been rigorously established; for a systematic survey of this family, we refer the reader to $[174,197]$. Most of Bug-family algorithms directly employ boundary following in close range as a hint to bypass an encountered obstacle. Motion toward the target is resumed as soon as a leaving condition is satisfied, which is designed to ensure global convergence and which verification often requires to memorize some prior sensory information. At the same time, these algorithms typically do not include any specific method of boundary following but instead take the capability of such following for granted. In this sense, they can be viewed as a higher level control strategies rather than completed methods of reactive navigation. What is more, practical implementation of this and other assumed capabilities (e.g., for instantaneous turns) not only may constitute a separate engineering problem but also may be impossible due to kinematic or dynamic constraints that cannot be ignored in practical setting. Implications of this impossibility basically lie in an uncharted territory.

A completed bug-type reactive navigation strategy (8) for target reaching with obstacle avoidance for a nonholonomic under-actuated and control-saturated vehicle (1) was proposed in ref. [178]. Provided that in (8), the sum of full turns of the target line of sight is reckoned in $\beta$, the reactive control law is shown to be capable of achieving the global robotics task: target reaching in a simply connected maze-like environment. This holds with the fixed turn direction in (d) (i.e., $\sigma$ in (8)) if the vehicle initial location $\boldsymbol{r}_{0}$ and the target $\boldsymbol{T}$ are not deep inside the maze. ${ }^{6}$ Otherwise, the claim is true with probability 1 if this direction is sometimes randomly updated. All these are valid if the vehicle is maneuverable enough to cope with the narrows and contortions of the maze, with constructive conditions for this being provided. Limited randomization permits to resolve navigational limit cycles without violation of the reactive nature of the overall algorithm. A number of deterministic methods to prevent these cycles are known (see, e.g., [205] and the above literature on the Bug-type algorithms), however they hardly can be classified as purely reactive.

4.1.4. Full information-based. In situations where more information about the obstacle is available, a clearer view of the immediate environment can be recreated. This means more informed navigation decisions may be able to be made. This can lead to desirable behaviors, such as variable speed and

${ }^{6}$ This means that neither of them lies in a cave of $d_{\uparrow}$-neighborhood $\mathcal{D}:=\left\{\boldsymbol{r}: \boldsymbol{d i s t}_{D}[\boldsymbol{r}] \leq d_{\uparrow}\right\}$, where $d_{\downarrow}$ is the triggering threshold from (8). The cave is defined as an area limited by a segment $\left[\boldsymbol{r}_{\diamond,}, \boldsymbol{r}_{*}\right]$ of a ray emitted from $\boldsymbol{T}$ such that $\boldsymbol{r}_{\diamond}, \boldsymbol{r}_{*} \in \partial \mathcal{D}$ and $\left(\boldsymbol{r}_{\diamond}, \boldsymbol{r}_{*}\right) \cap \partial \mathcal{D}=\emptyset$ and the smallest of two arcs of $\partial \mathcal{D}$ with the end points $\boldsymbol{r}_{\diamond}, \boldsymbol{r}_{*}$. 
offset distance from the obstacle. It also allows us to loosen some of the assumptions on the obstacle shape and curvature. An example of such behavior may be slowing down at concavities of a boundary and speeding up otherwise, or completely skipping concavities of sufficiently small size that serve only to introduce singularities into the motion. ${ }^{125,181}$

One such approach using abstract obstacle information is the VisBug class of algorithms, which navigates toward a visible edge of an obstacle inside the detection range (see, e.g., $[140,160,197])$. However, these algorithms are concerned with the overall strategy, and are not concerned with details relating to vehicle kinematics or the sensor model. Several approaches have been able to account for the vehicle dynamics, but still have inadequate models of the vehicle sensor. For example, in ref. [251] the joggers problem was proposed, which ensures safe navigation by ensuring the vehicle can stop in the currently sensed obstacle-free set. However, an abstract sensor model was used, which presumes the vehicle has continuous knowledge about the obstacle set. In ref. [92], a formulation achieves boundary following by picking instant goals based on observable obstacles. A ray-based sensor model is used, though a velocity-controlled holonomic model is assumed. In ref. [93], instant goals are also used, and allowance is made for the vehicle kinematics, however a ray-based obstacle sensor model was not used.

An MPC-based approach to boundary following has been proposed, which generates avoidance constraints and suitable target points to achieve boundary following. ${ }^{111}$ This was found to give better performance than existing methods when applied to acceleration-constrained vehicles, and may be a first step to applying MPC to the boundary following problems.

\subsection{Sensor-based path planning}

Trajectory planning using only sensor information was originally termed the joggers problem, since the vehicle must always maintain a path that brings it to a halt within the currently sensor area, see, e.g., $[8,251]$.

The classic dynamic window (see, e.g., [83, 201, 202]) and curvature velocity method (see, e.g., $[76,246])$ can be interpreted as a planning algorithm with a prediction horizon of a single time step. ${ }^{203}$ To this end, the range of considered control inputs is limited to those bringing the vehicle to a halt within the sensor visibility area, using only circular paths. This can also be easily extended to other vehicle shapes and models, ${ }^{241}$ also, measures are available, which reduce oscillatory behavior. ${ }^{262} \mathrm{~A}$ wider range of possible trajectory shapes has also been considered. ${ }^{31}$ The Lane-Curvature Method (see, e.g., [127]), and the Beam-Curvature Method (see, e.g., [76, 246]) are both variants based on a different trajectory selection process from a similar class of possible trajectories.

In all these cases, the justification for collision avoidance is based on essentially the same argument (the vehicle can stop while moving along the chosen trajectory). The differences in performance are mainly heuristic.

Approaches similar to the dynamic window have also been extended to cases where disturbance is present, using an approach similar to tube MPC. ${ }^{109}$ In addition, navigation systems that generate obstacle constraints by processing information from a ray-based sensor model have been proposed. ${ }^{107,111}$

MPC-type approaches have previously been used to navigate vehicles in unknown environments, see, e.g., [36, 130, 300]. Here, the MPC algorithm is combined with some type of mapping algorithm, however some of the rigorous guarantees normally provided in MPC approaches are harder to show. Robust MPC may also be used in unknown environments, see, e.g., [109]. However, there appears to be more room for more research in this particular area.

An interesting approach to collision avoidance using these types of methods is to estimate obstacle positions based on bearing measurements combined with some state estimation method. ${ }^{304}$ In this case, observability constraints can be taken into account during planning.

When compared to potential field methods, MPC methods generally perform better as they consider a more optimal path that plans ahead as obstacles are approached. They are also less conservative, bringing the robot closer to the edge of its control capability.

\subsection{Other reactive methods}

In this section, we describe methods that do not explicitly generate a path that moves around obstacles, without explicitly performing boundary following. 
Many approaches to this particular problem assume holonomic velocity-controlled vehicles. However, in this case, it is not a severe limitation, as methods for extending such methods to arbitrary dynamics—including acceleration-constrained vehicles—are available, see, e.g., [31, 189, 192]. This is based on transformations that provide a zone around the vehicle that essentially accounts for perturbations introduced by the dynamics, and may be applied to a range of navigation approaches. This means that the use of the simple model is of a lesser concern. Alternatively, a method has been proposed, which guarantees collision avoidance by ensuring that the distance to obstacles is always greater than the stopping distance. ${ }^{168}$ This approach may be useful in cases where little is known about the vehicle model.

4.3.1. Artificial potential field methods. A classic approach to reactive collision avoidance is to construct a virtual potential field that causes repelling from obstacles and attraction to the target. These are termed as APF methods, and this continues to be an active area of research. Note these are different from APF-based path planning methods-in this section, methods are considered, which compute the control input on-line based on the available information. Several improvements are listed as follows:

- Unicycle kinematics. Performance can be improved on vehicles with unicycle-type kinematics. Specifically, this can be achieved by moving the vehicles' reference point slightly away from the center of the vehicle, see, e.g., [220, 282].

- Local minima avoidance. The shape of the potential field can be designed to flow around obstacle concavities; some of these are termed harmonic potential fields and provide better performance with local minima (though these are impossible to fully solve deterministically using reactive algorithms), see, e.g., [171, 172].

- Closed loop performance. Alteration to the shape of the potential field leads to an improvement to the closed loop performance, see, e.g., [52, 124]. Additionally, reductions of oscillation in narrow corridors may be achieved, see, e.g., [219, 220]. However, in general, the closed loop trajectories of APF-based methods would not be optimal.

- Limited obstacle information. Examples are available where only the nearest obstacle point is available. ${ }^{45}$ Approaches that assume global knowledge about the workspace would not be suitable for sensor-based navigation.

- Actuator constraints. Examples that focus on satisfying actuator constraints are also available, see, e.g., $[88,155]$. However, these methods do not generally directly achieve acceleration bounds.

APF methods have lower computational requirements than local planning approaches, but this is becoming less of a concern with ever increasing computational powers of unmanned vehicles.

4.3.2. Uncategorized approaches. There are many other approaches that achieve collision avoidance, which do not fit into the above categories:

- The safe maneuvering zone is suited for kinematic unicycle model with saturation constraints, when the nearest obstacle point is known. ${ }^{142}$ This is somewhat similar to the deformable virtual zone, where the navigation is based on a function of obstacle detection ray length, ${ }^{143}$ though collision avoidance is not explicitly proven.

- The vector field histogram directs the vehicle toward sufficiently large gaps between detection rays. ${ }^{278}$ The Nearness Diagram is an improved version that employs a number of behaviors for a number of different situations, providing good performance even in particularly cluttered environments (see, e.g., [190, 191]).

- A method of probabilistically convergent on-line navigation involves randomly choosing tangents to travel down (see, e.g., [237]), or by use of the deterministic TangentBug algorithm (see, e.g., [119]). Tangent events can be detected from a ray-based sensor model (see, e.g., [246]) or by processing data from a camera sensor (see, e.g., [113]). This results in an abstract tangent sensor that reports the angle to tangents around the vehicle. A common method of achieving obstacle avoidance is to maintain a fixed angle between the tangent and the vehicles motion, see, e.g., [113, 245].

- A collision avoidance system based on MPC has been proposed and shown to successfully navigate real-world helicopters in unknown environments based on the nearest obstacle point within the 
visibility radius. ${ }^{248}$ However, this is less concerned with planning safe trajectories, but rather more with controlling vehicle dynamics.

- A different class of navigation laws is based on the Voronoi Diagram, which essentially describes the set of points equidistant from adjacent obstacles. In general, it leads to longer paths than the tangent graph, though it represents the smallest set of trajectories that span the free space in an environment. Navigation laws have been developed to equalize the distance to obstacles, when a velocity-controlled unicycle kinematic model is assumed (see, e.g., [287]).

- A method has been proposed, which considers the set of velocities that avoid obstacles without performing explicit path planning. ${ }^{286}$ This uses linear-quadratic-Gaussian (LQG) to account for uncertainty and is applicable to various types of linear vehicle models.

\section{Moving Obstacles}

Certain types of autonomous vehicle will unavoidably encounter moving obstacles, which are generally more challenging to avoid than static equivalents. The main factors that affect the difficulty of this problem are the characterization of the possible actions another object might take; the increased complexity of the search space and terminal constraints in the case of path planning; and additional conservativeness in the case of sensor-based systems.

At one extreme, an obstacle translating at a constant speed and in a constant direction may be accounted for by merely considering the future position of the obstacle. The other extreme is an obstacle pursuing the vehicle, for which the set of potential locations grows polynomially along the planning horizon. Several offerings also describe integrated approaches, including obstacle motion estimation from LiDAR sensors. ${ }^{194}$ However, in this section, discussion is focused on the avoidance behavior.

General planning algorithms suited for dynamic environments are also available, however in the absence of obstacle assumptions it is impossible to guarantee existence of a viable path, see, e.g., [94]. When planning in known environments, states that necessarily lead to collision- the Inevitable Collision States (ICS) - may also be abstracted and used to assist planning. ${ }^{212}$ If the motion of vehicles is known stochastically, the overall probability of collision for a probational trajectory may also be computed based on the expected behavior of other obstacles, see, e.g., [7].

\subsection{Human-like obstacles}

Several works attempt to characterize the motion of moving obstacles. For avoiding humans, several models of socially acceptable pedestrian behavior are available (see, e.g., [81, 203, 254, 314]). An approach that avoids obstacles based on the concept of personal space has been proposed and works well in practice. ${ }^{204}$ Other approaches that can avoid human-like obstacles while also considering the reciprocal effect of the vehicles motion have also been proposed. ${ }^{81,314}$

\subsection{Known obstacles}

Obstacles translating at a constant speed and in a constant direction may be avoided using the concept of a velocity obstacle, see, e.g., [78, 79, 247]. This is essentially the set of vehicle velocities that will result in collision with the obstacle, and by avoiding these velocities, collisions may be avoided. This result may be extended to arbitrary (but known) obstacle paths and more complex vehicle kinematics using the nonlinear velocity obstacle, see, e.g., [144]. The velocity obstacle method also extends to three-dimensional spaces, see, e.g., [249, 301].

\subsection{Kinematically constrained obstacles}

When obstacles are only known to satisfy nominal kinematic constraints, the set of possible obstacle positions grows drastically over time.

5.3.1. Path-based methods. There are three basic methods of planning trajectories, which avoid such obstacles:

- Ensuring that whenever a collision could possibly occur, the vehicle is stationary-this is referred to as passive motion safety (see, e.g., [20,35]). In some situations, it is impossible to show any higher form of collision avoidance, though it ultimately relies on the behavior of obstacles to avoid collisions. 
- Ensuring that the vehicle can move arbitrarily far away from the obstacle over a infinite horizon. This is discussed in ref. [284], in which the time minimal paths were calculated. Similar examples of approaches include more allowance for other uncertainties, see, e.g., [69].

- Ensuring that the vehicle lies in a set of points that cannot be easily reached by the obstacle. This is proposed in ref. [298], where under certain assumptions a non-empty set of points may be found, which lies just behind the obstacles movement direction. This allows avoidance over a infinite horizon, while being possibly less conservative than the previous option.

When performing path planning in a sensor-based paradigm, the main additional assumption is that any occluded part of the workspace must be considered as a potential dynamic obstacle..$^{35,50}$ Naturally, this makes the motion of any vehicles even more conservative.

5.3.2. Reactive methods. When moving obstacles are present in the workspace, it is still possible to design reactive navigation strategies that can provably prevent collisions, at least with some more restrictive assumptions about the obstacles' motion. These methods are outlined as follows:

- When obstacles are sufficiently spaced (so that multiple obstacles must not be simultaneously avoided), an extension of the velocity obstacle method has been designed to prevent collisions. ${ }^{294}$ This effectively steers the vehicle toward the projected edge of the obstacle.

- Some APF methods have been extended to moving obstacles, though without rigorous justification, see, e.g., [91, 220].

- Certain sliding-mode boundary following techniques reported in Section 4.1.2 have been successfully extended to handle moving obstacles with provable collision avoidance, assuming that the obstacles are sufficiently spaced and their motion and/or deformation satisfy some technical smoothness constraints, see, e.g., [185, 240].

In particular, the paper ${ }^{185}$ examines the navigation strategy proposed in ref. [181]. We recall that it consists in properly switching between moves to the target in straight lines, when possible, and sliding-mode-based bypassing en-route obstacles at a pre-specified distance by applying the slidingmode control law (3). Whereas the convergence and performance of this algorithm were demonstrated in ref. [181] only for static scenes with convex obstacles, [185] has demonstrated the viability of this strategy for the vehicle (1) traveling in dynamic environments cluttered with arbitrarily shaped obstacles. They are not assumed to be rigid or even solid: they are continuums of arbitrary and time-varying shapes undergoing general displacements, including rotations and deformations. Since the strategy includes a controller that ensures patrolling of the boundary of a moving domain at a pre-specified distance, the proposed solution can be used for pure border surveillance, which is of self-interest. It should be noted that the theoretical guarantees given in ref. [185] concern only boundary following problem by proving non-local collision-free convergence of the vehicle to the pre-specified distance to the moving and deforming boundary. Global target reaching in cluttered environments was illustrated by computer simulations and experiments with a real wheeled robot.

In ref. [293], the results of [185] on target reaching with obstacle avoidance were extended on a planar differential drive robot, exemplified by an automated intelligent wheelchair. This robot has two independently actuated driving wheels mounted on the same axle and maybe castor wheels, which do not affect its mobility. The driving wheels roll without sliding. The robot is controlled by the angular velocities $\omega_{l}$ and $\omega_{r}$ of the left and right driving wheels, respectively, which are limited by a common and given constant $\Omega$. Instead of (1), the relevant mathematical model of kinematics of the robot is as follows:

$$
\begin{array}{lll}
\dot{x}=v \cos \theta, & v=\frac{v_{l}+v_{r}}{2} & x(0)=x_{0}, \\
\dot{y}=v \sin \theta, & u=\frac{v_{r}-v_{l}}{2 L}, & y(0)=y_{0}, \\
\dot{\theta}=u & v_{i}=R_{w} \omega_{i}, & \theta(0)=\theta_{0}
\end{array}
$$

where $R_{w}$ is the radius of the driving wheels, $2 L$ is the length of the axle, and $\omega_{i}=\omega_{i}(t) \in$ $[-\Omega, \Omega], i=l, r$. Without any loss of generality, $v$ and $u$ can be treated as control variables. They uniquely determine the rotational velocities $\omega_{r}=(v+L u) / R_{w}, \omega_{l}=(v-L u) / R_{w}$ and obey the bound $|v|+L|u| \leq V:=R_{w} \Omega$. This bound implies restrictions on the forward and rotational movements of the robot. In particular, its speed cannot exceed $V$, and for given $v \in(-V, V)$, the turning radius of the robot is bounded from below by $R=\frac{L|v|}{V-|v|}$. 
The following extension of the obstacle avoidance strategy (3) is employed:

$$
\begin{aligned}
& u(t)=\frac{V-v(t)}{L} \cdot \operatorname{sign}\left\{\dot{d}(t)+\chi\left[d(t)-d_{0}\right]\right\}, \\
& v(t)=\Upsilon[d(t)]
\end{aligned}
$$

where $\chi(\cdot)$ is still the linear function with saturation and the smooth function $\Upsilon(\cdot):[0, \infty) \rightarrow(0, V)$ determines the robot's longitudinal speed depending on the current distance to the obstacle. The function $\Upsilon(\cdot)$ smoothly varies between two speeds $v_{0}$ and $v_{\text {cr }}$, i.e., $\Upsilon(d)=v_{0} \forall d \leq d_{0}^{\Upsilon}, \Upsilon(d)=$ $v_{\mathrm{cr}} \forall d \geq d_{\mathrm{cr}}>d_{0}^{\Upsilon}$. The speed $v_{0} \in(0, V)$ is used when bypassing obstacles, so $d_{0}<d_{0}^{\Upsilon}$; the larger $v_{\mathrm{cr}} \in\left(v_{0}, V\right)$ "cruise" speed is employed where there is no collision threat.

The control law (10) is activated in a close proximity of en-route obstacles. Whenever the robot is far away from them, it is driven toward the target in a straight line:

$$
u(t) \equiv 0, \quad v(t)=\Upsilon[d(t)] .
$$

Switching (11) $\mapsto(10)$ occurs when the distance to the nearest obstacle does not exceed a given threshold $d_{\text {av }} \in\left(d_{0}, d_{0}^{\Upsilon}\right]$ and $\dot{d}+\chi\left(d-d_{0}\right) \leq 0$; switching (10) $\mapsto(11)$ occurs when the wheelchair is oriented toward the target and $\dot{d}+\chi\left(d-d_{0}\right) \geq 0$.

Like in ref. [185], obstacles are continuums of arbitrary and time-varying shapes undergoing general displacements, including rotations and deformations. The main theoretical result guarantees non-local collision-free convergence of the robot to the desired distance $d_{0}$ to the moving and deforming boundary with always respecting a given safety margin $d_{\text {safe }}$ provided that some technical and partly unavoidable assumptions are satisfied. Global convergence to the target is illustrated via computer simulations and experiments with a real wheelchair. Furthermore, the algorithm of [185] was successfully applied to the problem of collision-free navigation of intelligent robotic hospital beds for critical neurosurgery patients. ${ }^{292}$

A quite simple and computationally efficient biologically inspired algorithm of collision-free navigation among moving obstacle was proposed in ref. [240]. The proposed navigation strategy is based on switching between moving to the target along straight lines, when possible, and a slidingmode obstacle avoidance navigation law. Mathematically rigorous analysis of the algorithm was given for the case of round obstacles moving with constant velocities. Computer simulations and experiments with a real robot show that the algorithm outperforms some well-known other methods such as APF and velocity obstacle-based navigation laws.

\section{Multiple Vehicle Navigation}

Navigation of multiple vehicle systems has gained much interest in recent years. As autonomous vehicles are used in greater concentrations, the probability of multiple vehicle encounters correspondingly increases, and new methods are required to avoid collision. A typical scenario is shown in Fig. 3.

The study of decentralized control laws for groups of mobile autonomous robots has emerged as a challenging new research area in recent years (see, e.g., [234, 290], and references therein). Broadly speaking, this problem falls within the domain of decentralized control, but the unique aspect of it is that groups of mobile robots are dynamically decoupled, meaning that the motion of one robot does not directly affect that of the others. This type of systems is viewed as a networked control system, which is an active field of research. For examples of more generalized work in this area, see, e.g., $[179,180,235,236]$. One of the important applications of navigation of multi-vehicle systems is sensing coverage. To improve coverage and reduce the cost of deployment in a geographically vast area, employing a network of mobile sensors for the coverage is an attractive option. Three types of coverage problems for robotics were studied in recent years: blanket coverage, ${ }^{238}$ barrier coverage, ${ }^{46,47}$ and sweep coverage. ${ }^{46,48}$ Combining existing coverage algorithms with effective collision avoidance methods is an open practically important problem.

While there is an extensive literature on centralized navigation of multiple vehicles, it is only briefly mentioned here, since it is generally not applicable to arbitrarily scalable on-line collision avoidance systems. Examples of off-line path planning systems, which can find near optimal trajectories for a set of vehicles are available, see, e.g., $[151,256]$. Another variation of this problem involves a 


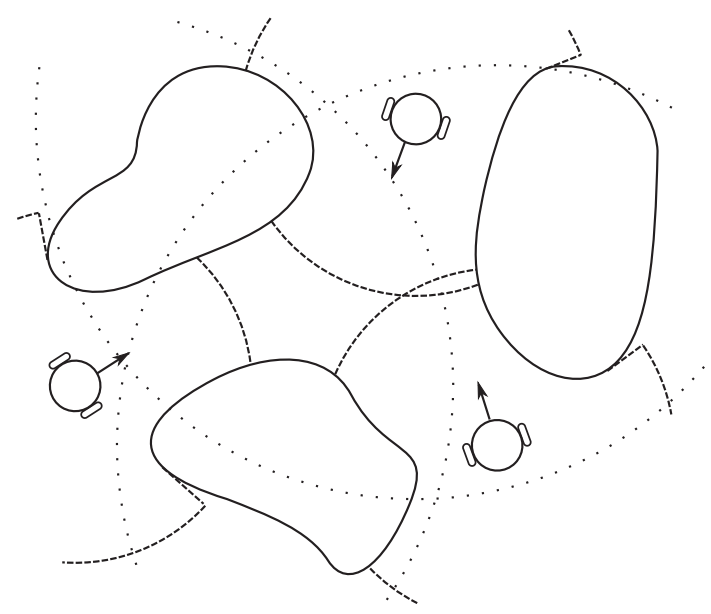

Fig. 3. Diagram of the multi-vehicle collision avoidance problem. This includes communication, sensing, and dynamic constraints.

precomputed prescription of the paths to be followed, where the navigation law must only find an appropriate velocity profile that avoids collisions (see, e.g., [56, 209]).

One of the issues facing navigation systems for multi-vehicle problems is deadlock and livelock. In the broadest definition, deadlock occurs when vehicles do not converge to their destinations, and instead converge to an arrangement that cannot be resolved using the nominal controller. Similarly, the term livelock means the vehicles continue to move indefinitely, but without converging to their respective destinations. These are situations that are very non-trivial to solve; however certain methods are currently able to provably achieve target convergence.

\subsection{Communication types}

There are three common modes of communication in multiple vehicle collision avoidance systems:

- Direct state measurement. This can be achieved using only sensor information to measure the state of the surrounding vehicles, and is used in many non-path-based reactive approaches.

- Single direction broadcasting. In addition to the physical state of the vehicle, additional variables are also transmitted, usually relating to the current trajectory of the vehicle. This is often used in mutual exclusion-based path constraints, where the projected states of other vehicles are avoided during planning, and is occasionally referred to as sign board communication.

- Two way communication. This can range from simple acknowledgment signals to full decentralized optimization algorithms. These are commonly used for decentralized MPC, though some MPC variants have been proposed where sign boards are sufficient.

A number of different models of communication delay and error are considered in networked navigation problems. The ability to cope with unit communication delays, packet dropouts, and finite communication ranges is definitely desirable in any navigation system.

\subsection{Reactive methods}

The most basic form of this problem only considers a small number of vehicles with at most a single point obstacle. For example, navigation laws have been proposed to avoid collisions between two vehicles traveling at constant speed with turning rate constraints, see, e.g., [86, 269]. A common example of this type of systems is an air traffic controller (ATC). However, these types of navigation systems do not directly relate to avoiding collisions in cluttered environments. ${ }^{132}$

6.2.1. Potential field methods. Potential field methods may be constructed to repel other vehicles. In some ways this approach is more satisfactory than the equivalent methods applied to static obstacles-for example, local minima are less of an issue in the absence of contorted obstacle shapes. Methods have been proposed, which avoid collision between an unlimited number of velocity-controlled unicycles or velocity-controlled linear vehicles. ${ }^{105,173,263}$ One variant, termed the 
multi-vehicle navigation function, is able to show convergence to targets in the absence of obstacles. However, these still use similar types of repulsive and attractive fields, see, e.g., [67, 268, 297].

Other variants also include measures to provably maintainable cohesion between groups, see, e.g., [66], while others have also been applied to vehicles with limited sensing capabilities. ${ }^{65}$ Navigation function methods have also been combined with MPC; this supplies some type of planning into the future to reject disturbances. ${ }^{227}$ Navigation function methods have also been applied to threedimensional scenarios. ${ }^{226}$

In cases where finite acceleration bounds are present (but still without any nonholonomic constraints), a mutual repulsion-based navigation system with a more sophisticated avoidance function has been proven to avoid collisions for up to three vehicles. ${ }^{106}$ When more vehicles are present, it is possible to back-step the additional dynamics into a velocity-controlled model. ${ }^{155,225}$ Furthermore, recently proposed control methods can achieve collision avoidance for Lagrangian systems with bounded inputs. ${ }^{225}$

Some other methods provide good practical results, though without focusing on mathematical analysis of collision avoidance, see, e.g., [45, 72, 73]. Many of these methods can be extended to static obstacles, and these combined systems are achieved by the same avoidance functions as the single vehicle case, see, e.g., [155].

6.2.2. Reciprocal collision avoidance methods. Approaches termed reciprocal collision avoidance (RCA) achieve collision avoidance by assuming that each vehicle takes some of the responsibility for each pair-wise conflict, with the resulting constraints forming a set of viable velocities from which a selection can be made using linear programming, see, e.g., [258, 283]. Some interesting extensions have been proposed to the ORCA concept, for example, it has been applied to both nonholonomic vehicles and linear vehicles with acceleration constraints, while maintaining collision avoidance. ${ }^{257,258,285}$ The method may be extended to arbitrary vehicle models, as rigorous avoidance is achieved via the addition of a generic bounded-deviation path tracking system. ${ }^{6,216,258}$ These methods are also able to include collision avoidance of static obstacles, which easily integrates into the navigation framework.

This idea is somewhat similar to a previous method based on collision cones called implicit cooperation. ${ }^{1}$ Another method has also been proposed, which is based on collision cones, called distributed reactive collision avoidance (DRCA). This has the benefit of showing achievement of the vehicles' objective in limited situations, ensuring minimum speed constraints are met when global information is available, and showing robustness to disturbance. ${ }^{137,138}$

6.2.3. Hybrid logic approaches. For these approaches, discrete logic rules are used to coordinate vehicles. In most cases, this is achieved through segregation of the workspace into cells, which can each only hold one vehicle, see, e.g., [22, 95, 198, 221]. In these cases, collisions can be prevented by devising a scheme where two vehicles do not attempt to occupy the same cell simultaneously. Additionally, many methods of integrating this with control of the vehicle's dynamics have been proposed, see, e.g., [55].

In some approaches, the generation of cells may be on-line and ad hoc. This is useful when minimum speed constraints are present — the vehicles may be instructed to maintain a circular holding pattern, and then to shift their holding pattern appropriately when safe. In this case, some different possibilities for the shifting logic have been proposed, for example, based on vehicle priority, ${ }^{131}$ or traffic rules. ${ }^{208}$ These methods are able to show convergence to the desired states, thus avoiding deadlock.

\subsection{Decentralized MPC}

While optimal centralized MPC is theoretically able to coordinate groups of vehicles, the underlying optimization process is too complex for any scalable real time application. Examples of centralized MPC for multiple vehicle systems are available (see, e.g., [74]).

Decentralized variants of MPC in general do not specifically address the problem of deadlock. For example, in ref. [135] a distributed navigation system is proposed, which is able to plan near optimal solutions that robustly prevent collisions, and allow altruistic behavior between the vehicles that monotonically decrease the global cost function. However, this does not equate to deadlock avoidance, which can currently only be solved in general using discrete graph-based methods. One 
very simple approach has been proposed to avoid this problem without a graph abstraction, though it has several limitations. ${ }^{108}$

A review of general decentralized MPC methods is available, ${ }^{23}$ along with a review specific to vehicle navigation. ${ }^{250}$ There are currently four main methods of generating deconflicted trajectories that seem suitable for coordination of multiple vehicles:

- Decentralized optimization can find the near-optimal solution for a multi-agent system using dual decomposition to find a set of trajectories for the system of vehicles, see, e.g., [214, 264, 291]. While this is more efficient than centralized optimization, it requires many iterations of communication exchange between vehicles in order to converge to a solution. Other decentralized planning algorithms may also be effective, for example, decentralized random tree-based methods have been proposed, which allows alteration of neighbors' plans. ${ }^{62}$

- Other approaches have been proposed using multiplexed MPC (see, e.g., [136, 255]), and sequential decentralization (see, e.g., $[3,13,194])$. The robust control input for each vehicle may be computed by updating the trajectory for each vehicle sequentially, at least when they are close. While multiplexed MPC is suited to real time implementation, a possible disadvantage is path planning cannot occur simultaneously in two adjacent vehicles. However, the same framework has been extended to provide collision avoidance in vehicle formation problems. ${ }^{296}$

- Another possible solution is to require acknowledgment signals before implementing a possible trajectory, and has the benefit of not requiring vehicles to be synchronized. This method seems an effective solution, ${ }^{18,19}$ however interaction between vehicles may cause planning delays under certain conditions.

- Approaches also have been proposed, which permit single communication exchanges per control update. ${ }^{59,211,281}$ This is done by including a coherence objective to prevent the vehicles from changing its planned trajectory significantly after transmitting it to other vehicles. Another approach uses different types of constraints to avoid coherence objectives, though it only works with a limited class of planning algorithms. ${ }^{110}$

MPC may also easily include maintenance of objectives other than collision avoidance. For example, radio propagation models have been included in the path evaluation function, so that communication between vehicles is maintained. ${ }^{102,103}$

When compared to potential field-based methods, MPC more naturally accounts for obstacles and complex vehicle model. Preliminary results have shown MPC-based methods have better closed loop performance than potential field-based ones. ${ }^{110}$

\section{Conclusions}

In this paper, we have provided a review of a range of techniques related to the navigation of unmanned vehicles through cluttered environments, which can rigorously achieve collision avoidance for some given assumptions about the system. This continues to be an active area of research, and we highlight a number of channels where current approaches may be improved. We also consider approaches to avoiding collisions between multiple vehicles, along with approaches for avoiding collision among moving obstacles. In particular, we focus on reactive approaches based on local sensor information, which seems more difficult and relevant than global approaches where full knowledge of the environment is assumed. Finally, we highlight the virtues of sliding-mode control, MPC, and decentralized MPC-based approaches to this problem, especially over the commonly used APF methods. In general, MPC navigation methods are more optimal, allow for the future position of the vehicle, account for situations where multiple obstacles, vehicles and complex vehicle dynamics are concurrently present more naturally, and do not necessarily carry an excessive computational burden. Through not generally optimal, sliding-mode control-based navigation methods are robust to noise, and can be rigorously proven to be correct for a range of vehicle models and navigation tasks.

\section{Acknowledgment}

This work was supported by the Australian Research Council. 


\section{References}

1. Y. Abe and M. Yoshiki, "Collision Avoidance Method for Multiple Autonomous Mobile Agents by Implicit Cooperation," Proceedings of the IEEE/RSJ International Conference on Intelligent Robots and Systems, Vol. 3, Maui, HI, USA (2001) pp. 1207-1212.

2. P. Abichandani, G. Ford, H. Y. Benson and M. Kam, "Mathematical Programming for Multi-Vehicle Motion Planning Problems," Proceedings of the IEEE International Conference on Robotics and Automation, St Paul, MN, USA (2012) pp. 3315-3322.

3. S. Adinandra, E. Schreurs and H. Nijmeijer, "A Practical Model Predictive Control for a Group of Unicycle Mobile Robots," Proceedings of the 4th IFAC Conference on Nonlinear Model Predictive Control, Vol. 4, Leeuwenhorst, Netherlands (2012) pp. 472-477.

4. M. A. Ahmadi-Pajouh, F. Towhidkhah, S. Gharibzadeh and M. Mashhadimalek, "Path planning in the hippocampo-prefrontal cortex pathway: An adaptive model based receding horizon planner," Med. Hypotheses 68(6), 1411-1415 (2007).

5. A. Albagul and Wahyudi, "Dynamic Modeling and Adaptive Traction Control for Mobile Robots," Proceedings of the 30th Annual Conference of IEEE Industrial Electronics Society, Vol. 1, Busan, Korea (2004) pp. 614-620.

6. J. Alonso-Mora, A. Breitenmoser, P. Beardsley and R. Siegwart, "Reciprocal Collision Avoidance for Multiple Car-Like Robots," Proceedings of the 2012 IEEE International Conference on Robotics and Automation, St Paul, MN, USA (2012) pp. 360-366.

7. D. Althoff, J. Kuffner, D. Wollherr and M. Buss, "Safety assessment of robot trajectories for navigation in uncertain and dynamic environments," Auton. Robot. 32(3), 285-302 (2012).

8. J. C. Alvarez, A. Shkel and V. Lumelsky, "Accounting for Mobile Robot Dynamics in Sensor-Based Motion Planning: Experimental Results," Proceedings of the IEEE International Conference on Robotics and Automation, Vol. 3, Lueven, Belgium (1998) pp. 2205-2210.

9. S. B. Andersson, "Curve tracking for rapid imaging in AFM," IEEE Trans. Nanobiosci. 6(4), 354-361 (2007).

10. R. C. Arkin, "Motor schema based mobile robot navigation," Int. J. Robot. Res. 8(4), 92-112 (1989).

11. R. C. Arkin, "Behavior-based robot navigation for extended domains," Adapt. Behav. 1(2), 201-225 (1992).

12. L. Armesto, V. Girbes, M. Vincze, S. Olufs and P. Munoz-Benavent, "Mobile Robot Obstacle Avoidance Based on Quasi-Holonomic Smooth Paths," In: Advances in Autonomous Robotics (Lecture Notes in Computer Science), Vol. 7429 (G. Herrmann et al., eds.) (Springer, Berlin, Germany, 2012) pp. 244255.

13. F. Augugliaro, A. P. Schoellig and R. D'Andrea, "Generation of Collision-Free Trajectories for a Quadrocopter Fleet: A Sequential Convex Programming Approach," Proceedings of the IEEE/RSJ International Conference on Intelligent Robots and Systems, Algarve, Portugal (2012) pp. 19171922.

14. R. Balakrishna and A. Ghosal, "Modeling of slip for wheeled mobile robots," IEEE Trans. Robot. Autom. 11(1), 126-132 (1995).

15. D. J. Balkcom, P. A. Kavathekar and M. T. Mason, "Time-optimal trajectories for an omni-directional vehicle," Int. J. Robot. Res. 25(10), 985-999 (2006).

16. C. Barat and M. J. Rendas, "Benthic Boundary Tracking Using a Profiler Sonar," Proceedings of the IEEE/RSJ International Conference on Intelligent Robots and Systems, Vol. 1, Las Vegas, NV, USA (Oct. 2003) pp. 830-835.

17. D. Baronov and J. Baillieul, "Reactive Exploration Through Following Isolines in a Potential Field," Proceedings of the American Control Conference, New York, NY, USA (Dec. 2007) pp. 2141-2146.

18. K. E. Bekris, D. K. Grady, M. Moll and L. E. Kavraki, "Safe distributed motion coordination for secondorder systems with different planning cycles," Int. J. Robot. Res. 31(2), 129-150 (2012).

19. K. E. Bekris, K. I. Tsianos and L. E. Kavraki, "Safe and distributed kinodynamic replanning for vehicular networks," Mobile Netw. Appl. 14(3), 292-308 (2009).

20. F. Belkhouche, "Reactive path planning in a dynamic environment," IEEE Trans. Robot. 25(4), 902-911 (2009).

21. S. Belkhous, A. Azzouz, M. Saad, V. Nerguizian and C. Nerguizian, "A novel approach for mobile robot navigation with dynamic obstacles avoidance," J. Intell. Robot. Syst. 44(3), 187-201 (2005).

22. C. Belta, A. Bicchi, M. Egerstedt, E. Frazzoli, E. Klavins and G. J. Pappas, "Symbolic planning and control of robot motion [grand challenges of robotics]," IEEE Robot. Autom. Mag. 14(1), 61-70 (2007).

23. A. Bemporad and D. Barcelli, "Decentralized model predictive control," In: Lecture Notes in Control and Information Sciences, Vol. 406 (A. Bemporad, M. Heemels and M. Johansson, eds.) (Springer, London, 2010) pp. 149-178.

24. A. Bemporad, M. D. Marco and A. Tesi, "Sonar-based wall-following control of mobile robots," ASME J. Dyn. Syst. Meas. Control 122(1), 226-230 (2000).

25. S. Bereg and D. Kirkpatrick, "Curvature-bounded Traversals of Narrow Corridors," Proceedings of the 21st Annual Symposium on Computational Geometry, Pisa, Italy (2005) pp. 278-287.

26. M. Kemp, A. L. Bertozzi and D. Marthaler, "Multi-UUV Perimeter Surveillance," Proceedings of the IEEE/OES Autonomous Underwater Vehicles Conference, Sebasco, ME, USA (Jun. 2004) pp. 102107. 
27. E. Besada-Portas, L. de la Torre, J. M. de la Cruz and B. de Andres-Toro, "Evolutionary trajectory planner for multiple UAVs in realistic scenarios," IEEE Trans. Robot. 26(4), 619-634 (2010).

28. G. Bevan, H. Gollee and J. O'reilly, "Automatic lateral emergency collision avoidance for a passenger car," Int. J. Control 80(11), 1751-1762 (2007)

29. A. Bicchi, G. Casalino and C. Santilli, "Planning shortest bounded-curvature paths for a class of nonholonomic vehicles among obstacles," J. Intell. Robot. Syst. 16(4), 387-405 (1996).

30. L. Blackmore, M. Ono and B. C. Williams, "Chance-constrained optimal path planning with obstacles," IEEE Trans. Robot. 27(6), 1080-1094 (2011).

31. J.-L. Blanco, J. Gonzalez and J.-A. Fernandez-Madrigal, "Extending obstacle avoidance methods through multiple parameter-space transformations," Auton. Robots 24(1), 29-48 (2008).

32. N. W. Bode, A. J. Wood and D. W. Franks, "Social networks and models for collective motion in animals," Behav. Ecol. Sociobiol. 65(2), 117-130 (2011).

33. F. Bonin-Font, A. Ortiz and G. Oliver, "Visual navigation for mobile robots: A survey," J. Intell. Robot. Syst. 53(3), 263-296 (2008).

34. V. Boquete, R. Garcia, R. Barea and M. Mazo, "Neural control of the movements of a wheelchair," $J$. Intell. Robot. Syst. 25(3), 213-226 (1999).

35. S. Bouraine, T. Fraichard and H. Salhi, "Provably safe navigation for mobile robots with limited field-ofviews in dynamic environments," Auton. Robots 32(3), 267-283 (2012).

36. A. Brooks, T. Kaupp and A. Makarenko, "Randomised MPC-based Motion-Planning for Mobile Robot Obstacle Avoidance," Proceedings of the 2009 IEEE International Conference on Robotics and Automation, Kobe, Japan (2009) pp. 397-402.

37. E. Burian, D. Yoeger, A. Bradley and H. Singh, "Gradient Search with Autonomous Underwater Vehicle using Scalar Measurements," Proceedings of the IEEE Symposium on Underwater Vehicle Technology, Monterey, CA (Jun. 1996) pp. 86-98.

38. M. Caccia, R. Bono and G. Bruzzone, "Variable-configuration UUVs for marine science applications," IEEE Robot. Autom. Mag. 6(2), 22-32 (1999).

39. J. M. Camhi and E. N. Johnson, "High-frequency steering maneuvers mediated by tactile cues: Antennal wall-following in the cockroach," J. Exp. Biol. 202(5), 631-643 (1999).

40. J. Canny, The Complexity of Robot Motion Planning (MIT Press, Cambridge, MA, 1988).

41. R. Carelli and E. O. Freire, "Corridor navigation and wall-following stable control for sonar-based mobile robots," Robot. Auton. Syst. 45(12), 235-247 (2003).

42. D. W. Casbeer, D. B. Kingston, R. W. Beard, T. W. McLain, S. M. Li and R. Mehra, "Cooperative forest fire surveillance using a team of small unmanned air vehicles," Int. J. Syst. Sci. 36(6), 351-360 (2006).

43. D. W. Casbeer, S. M. Li, R. W. Beard, T. W. McLain and R. K. Mehra, "Forest Fire Monitoring Using Multiple Small UAVs," Proceedings of the 2005 American Control Conference, Vol. 5, Minneapolis, MA, USA (2005) pp. 3530-3535.

44. A. Chakravarthy and D. Ghose, "Obstacle avoidance in a dynamic environment: A collision cone approach,” IEEE Trans. Syst. Man Cybern. 28(5), 562-574 (1998).

45. D. E. Chang, S. C. Shadden, J. E. Marsden and R. Olfati-Saber, "Collision Avoidance for Multiple Agent Systems," Proceedings of the 42nd IEEE Conference on Decision and Control, Vol. 1, Maui, HI, USA (2003) pp. 539-543.

46. T. M. Cheng and A. V. Savkin, "Decentralized control for mobile robotic sensor network self-deployment: Barrier and sweep coverage problems," Robotica 29(2), 283-294 (2011).

47. T. M. Cheng and A. V. Savkin, "Self-deployment of mobile robotic sensor networks for multilevel barrier coverage," Robotica 30(4), 661-669 (2012).

48. T. M. Cheng, A. V. Savkin and F. Javed, "Decentralized control of a group of mobile robots for deployment in sweep coverage," Robot. Auton. Syst. 59(7-8), 497-507 (2011).

49. H. Chitsaz, S. M. LaValle, D. J. Balkcom and M. T. Mason, "Minimum wheel-rotation paths for differentialdrive mobile robots," Int. J. Robot. Res. 28(1), 66-80 (2009).

50. W. Chung, S. Kim, M. Choi, J. Choi, H. Kim, C-B. Moon and J-B. Song, "Safe navigation of a mobile robot considering visibility of environment," IEEE Trans. Ind. Electron. 56(10), 3941-3950 (2009).

51. J. Chunyu, Zh. Qu, E. Pollak and M. Falash, "Reactive Target-tracking Control with Obstacle Avoidance of Unicycle-type Mobile Robots in a Dynamic Environment," American Control Conference, Baltimore, MD (Jun. 2010) pp. 1190-1195.

52. S. Cifuentes, J. M. Giron-Sierra and J. Jimenez, "Robot navigation based on discrimination of artificial fields: Application to single robots," Adv. Robot. 26(5-6), 605-626 (2012).

53. J. Cochran and M. Krstic, "Nonholonomic source seeking with tuning of angular velocity," IEEE Trans. Autom. Control 54(4), 717-731 (2009).

54. L. Consolini and M. Tosques, "A path following problem for a class of non-holonomic control systems with noise," Automatica 41(6), 1009-1016 (2005).

55. R. V. Cowlagi and P. Tsiotras, "Hierarchical motion planning with dynamical feasibility guarantees for mobile robotic vehicles," IEEE Trans. Robot. 28(2), 379-395 (2012).

56. R. Cui, B. Gao and J. Guo, "Pareto-optimal coordination of multiple robots with safety guarantees," Auton. Robots 32(3), 189-205 (2012).

57. N. Dadkhah and B. Mettler, "Survey of motion planning literature in the presence of uncertainty: Considerations for UAV guidance,” J. Intell. Robot. Syst. 65(1), 233-246 (2012). 
58. J. De Schutter, T. De Laet, J. Rutgeerts, W. Decra, R. Smits, E. Aertbelian, K. Claes and H. Bruyninckx, "Constraint-based task specification and estimation for sensor-based robot systems in the presence of geometric uncertainty," Int. J. Robot. Res. 26(5), 433-455 (2007).

59. M. Defoort, A. Kokosy, T. Floquet, W. Perruquetti and J. Palos, "Motion planning for cooperative unicycletype mobile robots with limited sensing ranges: A distributed receding horizon approach," Robot. Auton. Syst. 57(11), 1094-1106 (2009).

60. M. Defoort, J. Palos, A. Kokosy, T. Floquet and W. Perruquetti, "Performance-based reactive navigation for non-holonomic mobile robots," Robotica 27(2), 281-290 (2009).

61. M. Deng, A. Inoue, Y. Shibata, K. Sekiguchi and N. Ueki, "An Obstacle Avoidance Method for Two Wheeled Mobile Robot," Proceedings of the 2007 IEEE International Conference on Networking, Sensing and Control (2007), pp. 689-692.

62. V. Desaraju and J. How, "Decentralized path planning for multi-agent teams with complex constraints," Auton. Robots 32(4), 385-403 (2012).

63. G. N. DeSouza and A. C. Kak, "Vision for mobile robot navigation: A survey," IEEE Trans. Pattern Anal. Mach. Intell. 2(24), 237-267 (2002).

64. R. Diankov and J. Kuffner, "Randomized Statistical Path Planning," Proceedings of the 2007 IEEE/RSJ International Conference on Robots and Systems, San Diego, CA, USA (2007) pp. 1-6.

65. D. V. Dimarogonas and K. J. Kyriakopoulos, "Decentralized navigation functions for multiple robotic agents with limited sensing capabilities," J. Intell. Robot. Syst. 48(3), 411-433 (2007).

66. D. V. Dimarogonas and K. J. Kyriakopoulos, "Connectedness preserving distributed swarm aggregation for multiple kinematic robots," IEEE Trans. Robot. 24(5), 1213-1223 (2008).

67. D. V. Dimarogonas, S. G. Loizou, K. J. Kyriakopoulos and M. M. Zavlanos, "A feedback stabilization and collision avoidance scheme for multiple independent non-point agents," Automatica 42(2), 229-243 (2006).

68. B. Douillard, D. Fox, F. Ramos and H. Durrant-Whyte, "Classification and semantic mapping of urban environments," Int. J. Robot. Res. 30(1), 5-32 (2011).

69. N. E. Du Toit and J. W. Burdick, "Robot motion planning in dynamic, uncertain environments," IEEE Trans. Robot. 28(1), 101-115 (2012).

70. L. E. Dubins, "On curves of minimal length with a constraint on average curvature and with prescribed initial and terminal positions and tangents," Am. J. Math. 79(3), 497-516 (1957).

71. H. Durrant-Whyte and T. Bailey, "Simultaneous localization and mapping: Part I," IEEE Robot. Autom. Mag. 13(2), 99-110 (2006).

72. S. W. Ekanayake and P. N. Pathirana, "Formations of robotic swarm: An artificial force based approach," Int. J. Adv. Robot. Syst. 6(1), 7-24 (2009).

73. F. Fahimi, C. Nataraj and H. Ashrafiuon, "Real-time obstacle avoidance for multiple mobile robots," Robotica 27(2), 189-198 (2009).

74. M. Farrokhsiar and H. Najjaran, "An Unscented Model Predictive Control Approach to the Formation Control of Nonholonomic Mobile Robots," Proceedings of the IEEE International Conference on Robotics and Automation, St Paul, MN, USA (2012) pp. 1576-1582.

75. S. Fazli and L. Kleeman, "Wall following and Obstacle Avoidance Results from A Multi-DSP Sonar Ring on a Mobile Robot," IEEE International Conference Mechatronics and Automation, Vol. 1, Niagara Falls, Canada (Jul. 2005) pp. 432-437.

76. J. L. Fernandez, R. Sanz, J. A. Benayas and A. R. Diaguez, "Improving collision avoidance for mobile robots in partially known environments: The beam curvature method," Robot. Auton. Syst. 46(4), 205-219 (2004).

77. A. Ferrara and M. Rubagotti, "Sliding Mode Control of a Mobile Robot for Dynamic Obstacle Avoidance Based on a Time-varying Harmonic Potential Field," ICRA 2007 Workshop: Planning, Perception and Navigation for Intelligent Vehicles, Rome, Italy (Apr. 2007).

78. P. Fiorini and Z. Shiller, "Time Optimal Trajectory Planning in Dynamic Environments," Proceedings of the IEEE International Conference on Robotics and Automation, Minneapolis, MN, USA (1996) pp. 1553-1558.

79. P. Fiorini and Z. Shiller, "Motion planning in dynamic environments using velocity obstacles," Int. J. Robot. Res. 17(7), 760-772 (1998).

80. G. Flierl, D. Grunbaum, S. Levin and D. Olson, "From individuals to aggregations: The interplay between behavior and physics," J. Theor. Biol. 196(4), 397-454 (1999).

81. A. Foka and P. Trahanias, "Probabilistic autonomous robot navigation in dynamic environments with human motion prediction," Int. J. Soc. Robot. 2(1), 79-94 (2010).

82. T. Fossen, Guidance and Control of Ocean Vehicles (Wiley, NY, 1994).

83. D. Fox, W. Burgard and S. Thrun, "The dynamic window approach to collision avoidance," IEEE Robot. Autom. Mag. 4(1), 23-33 (1997).

84. T. Fraichard, "Trajectory planning in a dynamic workspace: A state-time space approach," Adv. Robot. 13(1), 75-94 (1999).

85. T. Fraichard and H. Asama, "Inevitable Collision States. A Step Towards Safer Robots?" IEEE International Conference on Intelligent Robots and Systems, Vol. 1, Las Vegas, NV, USA (2003) pp. 388393.

86. A. Fujimori, M. Teramoto, P. N. Nikiforuk and M. M. Gupta, "Cooperative collision avoidance between multiple mobile robots," J. Robot. Syst. 17(7), 347-363 (2000). 
87. Y. Gabriely and E. Rimon, "CBUG: A quadratically competitive mobile robot navigation algorithm," IEEE Trans. Robot. 24(6), 1451-1457 (2008).

88. M. Galicki, "Collision-free control of an omni-directional vehicle," Robot. Auton. Syst. 57(9), 889-900 (2009).

89. S. Garrido, L. Moreno, D. Blanco and P. Jurewicz, "Path planning for mobile robot navigation using voronoi diagram and fast marching," Int. J. Robot. Autom. 2(1), 42-64 (2011).

90. S. S. Ge and Y. J. Cui, "New potential functions for mobile robot path planning," IEEE Trans. Robot. Autom. 16(5), 615-620 (2000).

91. S. S. Ge and Y. J. Cui, "Dynamic motion planning for mobile robots using potential field method," Auton. Robots 13(3), 207-222 (2002).

92. S. S. Ge, X. Lai and A. A. Mamun, "Boundary following and globally convergent path planning using instant goals," IEEE Trans. Syst. Man Cybern. 35(2), 240-254 (2005).

93. S. S. Ge, X. Lai and A. A. Mamun, "Sensor-based path planning for nonholonomic mobile robots subject to dynamic constraints," Robot. Auton. Syst. 55(7), 513-526 (2007).

94. T. Gecks and D. Henrich, "Sensor-based Online Planning of Time-optimized Paths in Dynamic Environments," In: Advances in Robotics Research (T. Krager and F. M. Wahl, eds.) (Springer, BerlinHeidelberg, 2009) pp. 53-63.

95. R. W. Ghrist and D. E. Koditschek, "Safe cooperative robot dynamics on graphs," SIAM J. Control Optim. 40(5), 1556-1575 (2002).

96. A. Girard, A. S. Howell and J. K. Hedrick, "Border Patrol and Surveillance Missions using Multiple Unmanned Air Vehicles," Proceedings of the 43th IEEE Conference on Decision and Control, Paradise Island, Bahamas (2004) pp. 620-625.

97. C. Goerzen, Z. Kong and B. Mettler, "A survey of motion planning algorithms from the perspective of autonomous UAV guidance," J. Intell. Robot. Syst. 57(1-4), 65-100 (2009).

98. M. Gomez, R. V. Gonzalez, T. Martinez-Marin, D. Meziat and S. Sanchez, "Optimal motion planning by reinforcement learning in autonomous mobile vehicles," Robotica 30(2), 159-170 (2012).

99. R. Gonzalez, M. Fiacchini, J. L. Guzman, T. Alamo and F. Rodriguez, "Robust tube-based predictive control for mobile robots in off-road conditions," Robot. Auton. Syst. 59(10), 711-726 (2011).

100. L. Gracia and J. Tornero, "Kinematic modeling and singularity of wheeled mobile robots," Adv. Robot. 21(7), 793-816 (2007).

101. L. Gracia and J. Tornero, "Kinematic modeling of wheeled mobile robots with slip," Adv. Robot. 21(11), 1253-1279 (2007).

102. A. Grancharova, E. I. Gratli and T. A. Johansen, "Distributed MPC-based Path Planning for UAVs Under Radio Communication Path Loss Constraints," Proceedings of the IFAC Conference on Embedded Systems, Computational Intelligence and Telematics in Control, Wurzburg, Germany (2012) pp. 254-259.

103. E. Gratli and T. Johansen, "Path planning for UAVs under communication constraints using SPLAT! and MILP," J. Intell. Robot. Syst. 65(1), 265-282 (2012).

104. W. E. Green and P. Y. Oh, "Optic-flow-based collision avoidance," IEEE Robot. Autom. Mag. 15(1), 96-103 (2008).

105. E. G. Hernandez-Martinez and E. Aranda-Bricaire, "Convergence and Collision Avoidance in Formation Control: A Survey of the Artificial Potential Functions Approach," In: Multi-Agent Systems-Modeling, Control, Programming, Simulations and Applications. InTech (F. Alkhateeb, E. Al Maghayreh and I. Abu Doush, eds) (2011).

106. G. M. Hoffmann and C. J. Tomlin, "Decentralized Cooperative Collision Avoidance for Acceleration Constrained Vehicles," Proceedings of the 47th IEEE Conference on Decision and Control, Cancun, Mexico (2008) pp. 4357-4363.

107. S. Horn and K. Janschek, "A Set-based Global Dynamic Window Algorithm for Robust and Safe Mobile Robot Path Planning," Proceedings of the 41 st International Symposium on Robotics and the 6th German Conference on Robotics Munich, Germany (2010) pp. 1-7.

108. M. Hoy, "Deadlock Resolution for Navigation of Wheeled Robots in Continuous State-space," Proceedings of the International Conference on Automation, Robotics, Control and Vision, Guangzhou, China (2012) pp. 130-135.

109. M. Hoy, A. S. Matveev, M. Garratt and A. V. Savkin, "Collision free navigation of an autonomous unmanned helicopter in unknown urban environments: Sliding mode and MPC approaches," Robotica 30(4), 537-550 (2012).

110. M. Hoy, A. S. Matveev and A. V. Savkin, "Collision free cooperative navigation of multiple wheeled robots in unknown cluttered environments," Robot. Auton. Syst. 60(10), 1253-1266 (2012).

111. M. Hoy and A. V. Savkin, "A method of boundary following by a wheeled mobile robot based on sampled range information," J. Intell. Robot. Syst. 72(3-4), 463-482 (2013).

112. L. Huang, "Wall-following control of an infrared sensors guided wheeled mobile robot," Int. J. Intell. Syst. Technol. Appl. 7(1), 106-117 (2009).

113. W. H. Huang, B. R. Fajen, J. R. Fink and W. H. Warren, "Visual navigation and obstacle avoidance using a steering potential function," Robot. Auton. Syst. 54(4), 288-299 (2006).

114. M. Innocenti, L. Pollini and D. Turra, "A fuzzy approach to the guidance of unmanned air vehicles tracking moving targets," IEEE Trans. Control Syst. Technol., 16(6), 1125-1137 (2008).

115. M. E. Jefferies and W. Yeap, eds., Robotics and Cognitive Approaches to Spatial Mapping, Vol. 38 (Springer, Berlin Heidelberg, 2008). 
116. A. Joshi, T. Ashley, Y. R. Huang and A. L. Bertozzi, "Experimental Validation of Cooperative Environmental Boundary Tracking with On-board Sensors," Proceedings of the American Control Conference, St Louis, MO, USA (Jun. 2009) pp. 2630-2635.

117. V. Kallem, A. T. Komoroski and V. Kumar, "Sequential composition for navigating a nonholonomic cart in the presence of obstacles," IEEE Trans. Robot. 27(6), 1152-1159 (2011).

118. I. Kamon, E. Rimon and E. Rivlin, “A range-sensor based navigation algorithm,” Int. J. Robot. Res. 17(9), 934-953 (1991).

119. I. Kamon, E. Rimon and E. Rivlin, "Tangentbug: A range-sensor-based navigation algorithm," Int. J. Robot. Res. 17(9), 934-953 (1998).

120. I. Kamon and E. Rivlin, "Sensory-based motion planning with global proofs," IEEE Trans. Robot. Autom. 13(6), 814-822 (1997).

121. S. Karaman and E. Frazzoli, "Sampling-based algorithms for optimal motion planning," Int. J. Robot. Res. 30(7), 846-894 (2011).

122. A. L. Bertozzi, M. Kemp and D. Marthaler, "Determining Environmental Boundaries: Asynchronous Communication and Physical Scales," In: Cooperative Control (V. Kumar, N. E. Leonard and A .S. Morse, eds.) (Springer Verlag, Berlin, 2004) pp. 25-42.

123. F. Kendoul, "Survey of advances in guidance, navigation, and control of unmanned rotorcraft systems," J. Field Robot. 29(2), 315-378 (2012).

124. D. H. Kim and S. Shin, "New repulsive potential functions with angle distributions for local path planning," Adv. Robot. 20(1), 25-48 (2006).

125. J. Kim, F. Zhang and M. Egerstedt, "Curve tracking control for autonomous vehicles with rigidly mounted range sensors," J. Intell. Robot. Syst. 56(2), 177-197 (2009).

126. S. Kim, J. Russel and K. Koo, "Construction robot path-planning for earthwork operations," J. Comput. Civ. Eng. 17(2), 97-104 (2003).

127. N. Y. Ko and R. G. Simmons, "The Lane-curvature Method for Local Obstacle Avoidance," Proceedings of the 1998 IEEE/RSJ International Conference on Intelligent Robots and Systems, Vol. 3, Victoria, Canada (1998) pp. 1615-1621.

128. S. Koenig and M. Likhachev, "Fast replanning for navigation in unknown terrain," IEEE Trans. Robot. 21(3), 354-363 (2005).

129. K. Kozlowski, Robot Motion and Control (Springer, London, 2009).

130. P. Krishnamurthy and F. Khorrami, "GODZILA: A low-resource algorithm for path planning in unknown environments," J. Intell. Robot. Syst. 48(3), 357-373 (2007).

131. A. Krontiris and K. E. Bekris, "Using Minimal Communication to Improve Decentralized Conflict Resolution for Non-holonomic Vehicles," Proceedings of the 2011 IEEE/RSJ International Conference on Intelligent Robots and Systems, San Francisco, CA, USA (2011) pp. 3235-3240.

132. J. K. Kuchar and L. C. Yang, "A review of conflict detection and resolution modeling methods," IEEE Trans. Intell. Trans. Syst. 1(4), 179-189 (2000).

133. R. Kulić and Z. Vukić, "Methodology of concept control synthesis to avoid unmoving and moving obstacles," J. Intell. Robot. Syst. 45(1), 267-294 (2006).

134. H. Kurniawati, Y. Du, D. Hsu and W. S. Lee, "Motion planning under uncertainty for robotic tasks with long time horizons," Int. J. Robot. Res. 30(3), 308-323 (2011).

135. Y. Kuwata and J. P. How, "Cooperative distributed robust trajectory optimization using receding horizon MILP," IEEE Trans. Control Syst. Technol. 19(2), 423-431 (2011).

136. Y. Kuwata, A. Richards, T. Schouwenaars and J. P. How, "Distributed robust receding horizon control for multivehicle guidance," IEEE Trans. Control Syst. Technol. 15(4), 627-641 (2007).

137. E. Lalish and K. Morgansen, "Distributed reactive collision avoidance," Auton. Robots 32(3), 207-226 (2012).

138. E. Lalish, K. A. Morgansen and T. Tsukamaki, "Decentralized Reactive Collision Avoidance for Multiple Unicycle-type Vehicles," Proceedings of the American Control Conference, Seattle, WA, USA (2008) pp. 5055-5061.

139. R. Langer, L. Coelho and G. Oliveira, "K-bug, A New Bug Approach for Mobile Robot's Path Planning," Proceedings of the IEEE International Conference on Control Applications, Singapore (Oct. 2007) pp. 403-408.

140. W. Langson, I. Chryssochoos, S. V. Rakovic and D. Q. Mayne, "Robust model predictive control using tubes," Automatica 40(1), 125-133 (2004).

141. L. Lapierre and B. Jouvencel, "Robust nonlinear path-following control of an AUV," IEEE J. Ocean. Eng. 33(2), 89-102 (2008)

142. L. Lapierre and R. Zapata, "A guaranteed obstacle avoidance guidance system," Auton. Robots 32(3), 177-187 (2012).

143. L. Lapierre, R. Zapata and P. Lepinay, "Combined path-following and obstacle avoidance control of a wheeled robot," Int. J. Robot. Res. 26(4), 361-375 (2007).

144. F. Large, C. Lauger and Z. Shiller, "Navigation among moving obstacles using the NLVO: Principles and applications to intelligent vehicles," Auton. Robots 19(2), 159-171 (2005).

145. J. C. Latombe, Robot Motion Planning (Kluwer Academic Publishers, London, 1991).

146. B. Lau, C. Sprunk and W. Burgard, "Kinodynamic Motion Planning for Mobile Robots Using Splines," Proceedings of the IEEE/RSJ International Conference on Intelligent Robots and Systems, St Louis, MO, USA (2009) pp. 2427-2433. 
147. S. L. Laubach and J. W. Burdick, "An Autonomous Sensor-Based Path-Planner for Planetary Microrovers," Proceedings of the IEEE International Conference on Robotics and Automation, Detroit, MI, USA (May 1999) pp. 347-354.

148. D. N. Lee, "Guiding movements by coupling taus," Ecol. Psychol. 10(3-4), 221-250 (1998).

149. H. Lee, V. I. Utkin and A. Malinin, "Chattering reduction using multiphase sliding mode control," Int. J. Control 82(9), 1720-1737 (2009).

150. K. B. Lee and M. H. Han, "Lane-following method for high speed autonomous vehicles," Int. J. Automot. Technol. 9(5), 607-613 (2008).

151. W. Li and C. G. Cassandras, "A cooperative receding horizon controller for multivehicle uncertain environments," IEEE Trans. Autom. Control 51(2), 242-257 (2006).

152. S. R. Lindemann, I. I. Hussein and S. M. LaValle, "Real Time Feedback Control for Nonholonomic Mobile Robots with Obstacles," Proceedings of the 45th IEEE Conference on Decision and Control, San Diego, CA, USA (Dec. 2006) pp. 2406-2411.

153. Y-H. Liu and S. Arimoto, "Path planning using a tangent graph for mobile robots among polygonal and curved obstacles," Int. J. Robot. Res. 11(4), 376-382 (1992).

154. M. A. Hsieh, S. Loizou and V. Kumar, "Stabilization of Multiple Robots on Stable Orbits via Local Sensing," Proceedings of the IEEE Conference on Robotics and Automation, Rome, Italy (Apr. 2007) pp. 2312-2317.

155. S. G. Loizou and K. J. Kyriakopoulos, "Navigation of multiple kinematically constrained robots," IEEE Trans. Robot. 24(1), 221-231 (2008).

156. A. S. Lopez, R. Zapata and M. A. Osorio-Lama, "Sampling-based motion planning: A survey," Comput. Sistem. 12(1), 5-24 (2008).

157. E. M. Low, I. R. Manchester and A. V. Savkin, "A biologically inspired method for vision-based docking of wheeled mobile robots," Robot. Auton. Syst. 55(10), 769-784 (2007).

158. V. Lumelsky and A. A. Stepanov, "Path-planning strategies for a point mobile automaton moving amidst unknown obstacles of arbitrary shape," Algorithmica 2(1), 403-430 (1987).

159. V. Lumelsky and S. Tiwari, "An Algorithm for Maze Searching with Azimuth Input," Proceedings of the IEEE Conference on Robotics and Automation, San Diego, CA, USA (May 1991) pp. 111-116.

160. V. J. Lumelsky and T. Skewis, "Incorporating range sensing in the robot navigation function," IEEE Trans. Syst. Man Cybern. 20(5), 1058-1069 (1990).

161. V. J. Lumelsky and A. A. Stepanov, "Dynamic path planning for a mobile automaton with limited information on the environment," IEEE Trans. Autom. Control 31(11), 1058-1063 (1986).

162. P. Maes and R. A. Brooks, "Learning to Coordinate Behaviors," Proceedings of the AAAI, Boston, MA (1990) pp. 796-802.

163. E. Magid and E. Rivlin, "Cautiousbug: A Competitive Algorithm for Sensor-based Robot Navigation," Proceedings of the IEEE/RSJ International Conference on Intelligent Robots and Systems, Sendai, Japan (Sep. 2004) pp. 2757-2762.

164. L. Magni, D. Raimondo and F. Allgower, Nonlinear Model Predictive Control: Towards New Challenging Applications (Springer-Verlag, Berlin, Germany, 2009).

165. M. Malisoff, F. Mazenc and F. Zhang, "Input-to-state Stability for Curve Tracking Control: A Constructive Approach," Proceedings of the American Control Conference, San Francisco, CA (USA, 2011) pp. 19841989.

166. I. R. Manchester and A. V. Savkin, "Circular navigation missile guidance with incomplete information and uncertain autopilot model," J. Guid. Control Dyn. 27(6), 1076-1083 (2004).

167. I. R. Manchester and A. V. Savkin, "Circular navigation guidance law for precision missile/target engagement," J. Guid. Control Dyn. 29(2), 1287-1292 (2006).

168. G. Manor and E. Rimon, "High-speed Navigation of a Uniformly Braking Mobile Robot Using PositionVelocity Configuration Space," Proceedings of the IEEE International Conference on Robotics and Automation, St Paul, MN, USA (2012) pp. 193-199.

169. D. Marthaler and A. L. Bertozzi, "Tracking Environmental Level Sets with Autonomous Vehicles," In: Recent Developments in Cooperative Control and Optimization (S. Butenko, R. Murphey and P. M. Pardalos, eds.), Vol. 3 (Kluwer, Boston, MA, 2003).

170. E. Masehian and Y. Katebi, "Robot motion planning in dynamic environments with moving obstacles and target," Int. J. Mech. Syst. Sci. Eng. 1(1), 20-25 (2007).

171. A. Masoud, "Kinodynamic motion planning," IEEE Robot. Autom. Mag. 17(1), 85-99 (2010).

172. A. Masoud, "A harmonic potential approach for simultaneous planning and control of a generic UAV platform," J. Intell. Robot. Syst. 65(1), 153-173 (2012).

173. S. Mastellone, D. M. Stipanovic, C. R. Graunke, K. A. Intlekofer and M. W. Spong, "Formation control and collision avoidance for multi-agent non-holonomic systems: Theory and experiments," Int. J. Robot. Res. 27(1), 107-126 (2008).

174. F. Mastrogiovanni, A. Sgorbissa and R. Zaccaria, "Robust navigation in an unknown environment with minimal sensing and representation," IEEE Trans. Syst. Man Cybern. 39(1), 212-229 (2009).

175. M. J. Mataric, "Behavior-based Control: Main Properties and Implications," Proceedings of the IEEE International Conference on Robotics and Automation Nice, France (1992) pp. 46-54.

176. A. S. Matveev, M. Hoy, J. Katupitiya and A. V. Savkin, "Nonlinear sliding mode control of an unmanned agricultural tractor in the presence of sliding and control saturation," Robot. Auton. Syst. 61(9), 973-987 (2013). 
177. A. S. Matveev, M. Hoy and A. V. Savkin, "The problem of boundary following by a unicycle-like robot with rigidly mounted sensors," Robot. Auton. Syst. 61(3), 312-327 (2013).

178. A. S. Matveev, M. C. Hoy and A. V. Savkin, "A method for reactive navigation of nonholonomic robots in maze-like environments," Automatica 49(5), 1268-1274 (2013).

179. A. S. Matveev and A. V. Savkin, "The problem of state estimation via asynchronous communication channels with irregular transmission times," IEEE Trans. Autom. Control 48(4), 670-676 (2003).

180. A. S. Matveev and A. V. Savkin, Estimation and Control over Communication Networks (Birkhauser, Boston, 2009).

181. A. S. Matveev, H. Teimoori and A. V. Savkin, "A method for guidance and control of an autonomous vehicle in problems of border patrolling and obstacle avoidance," Automatica 47(3), 515-514 (2011).

182. A. S. Matveev, H. Teimoori and A. V. Savkin, "Navigation of a unicycle-like mobile robot for environmental extremum seeking," Automatica 47(1), 85-91 (2011).

183. A. S. Matveev, H. Teimoori and A. V. Savkin, "Range-only measurements based target following for wheeled mobile robots," Automatica 47(1), 177-184 (2011).

184. A. S. Matveev, H. Teimoori and A. V. Savkin, "Method for tracking of environmental level sets by a unicycle-like vehicle," Automatica 48(9), 2252-2261 (2012).

185. A. S. Matveev, C. Wang and A. V. Savkin, "Real-time navigation of mobile robots in problems of border patrolling and avoiding collisions with moving and deforming obstacles," Robot. Auton. Syst. 60(6), 769-788 (2012).

186. D. Q. Mayne, E. C. Kerrigan, E. J. van Wyk and P. Falugi, "Tube-based robust nonlinear model predictive control," Int. J. Robust Nonlinear Control 21(11), 1341-1353 (2011).

187. D. Q. Mayne and S. Rakovic, "Model predictive control of constrained piecewise affine discrete-time systems," Int. J. Robust Nonlinear Control 13(3-4), 261-279 (2003).

188. A. Micaelli and C. Samson, "Trajectory tracking for unicycle-type and two-steering wheels mobile robots," Technical Report INRIA: Technical Report No: 2097, Institut national de recherche en informatique et en automatique (1993).

189. J. Minguez and L. Montano, "The Ego-Kinodynamic Space: Collision Avoidance for Any Shape Mobile Robots with Kinematic and Dynamic Constraints," Proceedings of the 2003 IEEE/RSJ International Conference on Intelligent Robots and Systems, Vol. 1, Las Vegas, NV, USA (2003) pp. 637-643.

190. J. Minguez and L. Montano, "Nearness diagram (ND) navigation: Collision avoidance in troublesome scenarios," IEEE Trans. Robot. Autom. 20(1), 45-59 (2004).

191. J. Minguez and L. Montano, "Sensor-based robot motion generation in unknown, dynamic and troublesome scenarios," Robot. Auton. Syst. 52(4), 290-311 (2005).

192. J. Minguez and L. Montano, "Extending collision avoidance methods to consider the vehicle shape, kinematics, and dynamics of a mobile robot," IEEE Trans. Robot. 25(2), 367-381 (2009).

193. P. Moghadam, W. S. Wijesoma and J. F. Dong, "Improving Path Planning and Mapping Based on Stereo Vision and Lidar," Proceedings of the International Conference on Control, Automation, Robotics and Vision, Hanoi, Vietnam (2008).

194. L. Montesano, J. Minguez and L. Montano, "Modeling dynamic scenarios for local sensor-based motion planning," Auton. Robots 25(3), 231-251 (2008).

195. D. Morgan, S.-J. Chung and F. Y. Hadaegh, "Decentralized Model Predictive Control of Swarms of Spacecraft Using Sequential Convex Programming," Proceedings of the AAS/AIAA Space Flight Mechanics Conference, Kauai, HI, USA (2013).

196. Y. K. Nak and R. Simmons, "The Lane-Curvature Method for Local Obstacle Avoidance," IEEE International Conference on Robotics and Automation, Vol. 3, Lueven, Belgium (Nov. 1998) pp. 16151621.

197. J. Ng, An Analysis of Mobile Robot Navigation Algorithms in Unknown Environments, Ph.D. Thesis, The University of Western Australia, Perth, Australia 2010.

198. J. Ng and T. Braunl, "Performance comparison of bug navigation algorithms," J. Intell. Robot. Syst. 50(1), 73-84 (2007).

199. T. Nishi, M. Ando and M. Konishi, "Distributed route planning for multiple mobile robots using an augmented lagrangian decomposition and coordination technique," IEEE Trans. Robot. 21(6), 1191-1200 (2005).

200. H. Noborio, "A sufficient condition for designing a family of sensor based deadlock free planning algorithms," Adv. Robot. 7(5), 413-433 (1993).

201. H. Noborio and T. Yoshioka, "An On-line and Deadlock-free Path Planning Algorithm Based on World Topology," Proceedings of the IEEE/RSJ Conference on Intelligent Robots and Systems, Yokohama, Japan (Dec. 1993) pp. $1425-1430$

202. P. Ogren and N. Leonard, "A Tractable Convergent Dynamic Window Approach to Obstacle Avoidance," Proceedings of IEEE International Conference on Intelligent Robots and Systems, Lausanne, Switzerland (2002) pp. 595-600.

203. P. Ogren and N. E. Leonard, "A convergent dynamic window approach to obstacle avoidance," IEEE Trans. Robot. 21(2), 188-195 (2005).

204. T. Ohki, K. Nagatani and K. Yoshida, "Local path planner for mobile robot in dynamic environment based on distance time transform method," Adv. Robot. 26(14), 1623-1647 (2012).

205. C. Ordonez, E. G. Collins, Jr., M. F. Selekwa and D. D. Dunlap, "The virtual wall approach to limit cycle avoidance for unmanned ground vehicles," Robot. Auton. Syst. 56(8), 645-657 (2008). 
206. E. Ostertag, "An improved path-following method for mixed h-2/h-infinity controller design," IEEE Trans. Autom. Control 53(8), 1967-1971 (2008).

207. E. Owen and L. Montano, "A Robocentric Motion Planner for Dynamic Environments Using the Velocity Space," IEEE International Conference on Intelligent Robots and Systems, Vol. 1, Beijing, China (2006) pp. 2833-2838.

208. L. Pallottino, V. G. Scordio, A. Bicchi and E. Frazzoli, "Decentralized cooperative policy for conflict resolution in multivehicle systems," IEEE Trans. Robot. 23(6), 1170-1183 (2007).

209. J. M. Park, D. W. Kim, Y. S. Yoon, H. J. Kim and K. S. Yi, "Obstacle avoidance of autonomous vehicles based on model predictive control," Proc. Inst. Mech. Eng. 223(12), 1499-1516 (2009).

210. J. Peng and S. Akella, "Coordinating multiple robots with kinodynamic constraints along specified paths," Int. J. Robot. Res. 24(4), 295-310 (2005).

211. W. Peng, D. Baocang and Z. Tao, "Distributed Receding Horizon Control for Nonholonomic Multi-vehicle System with Collision Avoidance," Proceedings of the 31st Chinese Control Conference, Hefei, China (2012) pp. 6327-6332.

212. S. Petti and T. Fraichard, "Partial Motion Planning Framework for Reactive Planning within Dynamic Environments," Proceedings of the AAAI International Conference on Advanced Robotics, Barcelona, Spain (2005).

213. Zh. Qu, J. Wang and C. E. Plaisted, "A new analytical solution to mobile robot trajectory generation in the presence of moving obstacles," IEEE Trans. Robot. 20(6), 978-993 (2004).

214. R. L. Raffard, C. J. Tomlin and S. P. Boyd, "Distributed Optimization for Cooperative Agents: Application to Formation Flight," Proceedings of the 43rd IEEE Conference on Decision and Control, Vol. 3, Paradise Island, Bahamas (2004) pp. 2453-2459.

215. S. V. Rakovic, E. C. Kerrigan, K. I. Kouramas and D. Q. Mayne, "Invariant approximations of the minimal robust positively invariant set," IEEE Trans. Autom. Control 50(3), 406-410 (2005).

216. A. T. Rashid, A. A. Ali, M. Frasca and L. Fortuna, "Multi-robot collision-free navigation based on reciprocal orientation," Robot. Auton. Syst. 60(10), 1221-1230 (2012).

217. J. A. Reeds and L. A. Shepp, "Optimal paths for a car that goes both forwards and backwards," Pac. J. Math. 145(2), 367-393 (1990).

218. J. Reif and M. Sharir, "Motion planning in the presence of moving obstacles," J. ACM 41(4), 764-790 (1994).

219. J. Ren, K. A. McIsaac and R. V. Patel, "Modified newton's method applied to potential field-based navigation for mobile robots," IEEE Trans. Robot. 22(2), 384-391 (2006).

220. J. Ren, K. A. McIsaac and R. V. Patel, "Modified Newton's method applied to potential field-based navigation for nonholonomic robots in dynamic environments," Robotica 26(1), 117-127 (2008).

221. S. A. Reveliotis and E. Roszkowska, "Conflict resolution in free-ranging multivehicle systems: A resource allocation paradigm," IEEE Trans. Robot. 27(2), 283-296 (2011).

222. A. Richards and J. P. How, "Robust Stable Model Predictive Control with Constraint Tightening," Proceedings of the American Control Conference, Minneapolis, MN, USA (2006) pp. 1557-1562.

223. A. Richards and J. P. How, "Robust variable horizon model predictive control for vehicle maneuvering," Int. J. Robust Nonlinear Control 16(7), 333-351 (2006).

224. A. Richards and J. P. How, "Robust distributed model predictive control," Int. J. Control 80(9), 1517-1531 (2007).

225. E. J. Rodriguez-Seda and M. W. Spong, "Guaranteed Safe Motion of Multiple Lagrangian Systems with Limited Actuation," Proceedings of the 51st IEEE Conference on Decision and Control, Maui, HI, USA (2012) pp. 2773-2780.

226. G. Roussos, D. V. Dimarogonas and K. J. Kyriakopoulos, "3d navigation and collision avoidance for nonholonomic aircraft-like vehicles," Int. J. Adapt. Control Signal Process. 24(10), 900-920 (2010).

227. G. P. Roussos, G. Chaloulos, K. J. Kyriakopoulos and J. Lygeros, "Control of Multiple Non-Holonomic Air Vehicles Under Wind Uncertainty Using Model Predictive Control and Decentralized Navigation Functions," Proceedings of the 47th IEEE Conference on Decision and Control, Cancun, Mexico (2008) pp. $1225-1230$.

228. M. Rubagotti, D. M. Raimondo, A. Ferrara and L. Magni, "Robust model predictive control with integral sliding mode in continuous-time sampled-data nonlinear systems," IEEE Trans. Autom. Control 56(3), 556-570 (2010).

229. G. M. Saggiani and B. Teodorani, "Rotary wing UAV potential applications: An analytical study through a matrix method," Aircr. Eng. Aerosp. Technol., Int. J. 76, 6-14 (2004).

230. C. Samson, "Control of chained systems: Application to path-following and time-varying point stabilization of mobile robots," IEEE Trans. Autom. Control 40, 64-77 (1995).

231. A. Sankaranarayanan and M. Vidyasagar, "Path Planning for Moving a Point Object Amidst Unknown Obstacles in a Plane: A New Algorithm and a General Theory for Algorithm Development," Proceedings of the IEEE International Conference on Decision and Control, Brighton, UK (Dec. 1991) pp. 11111119.

232. A. Sankaranarayanan and M. Vidyasagar, "A New Algorithm for Robot Curve-following Amidst Unknown Obstacles, and a Generalization of Maze-Searching," Proceedings of the IEEE International Conference on Robotics and Automation, Nice, France (May 1992) pp. 2487-2494. 
233. B. M. Sathyaraj, L. C. Jain, A. Finn and S. Drake, "Multiple UAVs path planning algorithms: A comparative study," Fuzzy Optim. Decis. Mak. 7(3), 257-267 (2008).

234. A. V. Savkin, "Coordinated collective motion of groups of autonomous mobile robots: Analysis of Vicsek's model," IEEE Trans. Autom. Control 49(6), 981-983 (2004).

235. A. V. Savkin, "Analysis and synthesis of networked control systems: Topological entropy, observability, robustness and optimal control," Automatica 42(1), 51-62 (2006).

236. A. V. Savkin and T. M. Cheng, "Detectability and output feedback stabilizability of nonlinear networked control systems," IEEE Trans. Autom. Control 52(4), 730-735 (2007).

237. A. V. Savkin and M. Hoy, "Reactive and the shortest path navigation of a wheeled mobile robot in cluttered environments," Robotica 31(2), 323-330 (2013).

238. A. V. Savkin, F. Javed and A. S. Matveev, "Optimal distributed blanket coverage self-deployment of mobile wireless sensor networks," IEEE Commun. Lett. 16(6), 949-951 (2012).

239. A. V. Savkin and H. Teimoori, "Bearings-only guidance of a unicycle-like vehicle following a moving target with a smaller minimum turning radius," IEEE Trans. Autom. Control 55(10), 2390-2395 (2010).

240. A. V. Savkin and C. Wang, "A simple biologically inspired algorithm for collision-free navigation of a unicycle-like robot in dynamic environments with moving obstacles," Robotica 31(6), 993-1001 (2013).

241. C. Schlegel, "Fast Local Obstacle Avoidance Under Kinematic and Dynamic Constraints for a Mobile Robot," Proceedings of the 1998 IEEE/RSJ International Conference on Intelligent Robots and Systems, Vol. 1, Victoria, Canada (1998) pp. 594-599.

242. E. Scholte and M. E. Campbell, "Robust nonlinear model predictive control with partial state information," IEEE Trans. Control Syst. Technol. 16(4), 636-651 (2008).

243. P. O. Scokaert and D. Q. Mayne, "Min-max feedback model predictive control for constrained linear systems," IEEE Trans. Autom. Control 43(8), 1136-1142 (1998).

244. M. Seder, K. Macek and I. Petrovic, "An Integrated Approach to Realtime Mobile Robot Control in Partially Known Indoor Environments," Proceedings of the 31 st Annual Conference of the IEEE Industrial Electronics Society, Raleigh, NC, USA (Nov. 2005) pp. 1785-1790

245. R. Sharma, J. Saunders and R. Beard, "Reactive path planning for micro air vehicles using bearing-only measurements," J. Intell. Robot. Syst. 65(1), 409-416 (2012).

246. C. Shi, Y. Wang and J. Yang, "A local obstacle avoidance method for mobile robots in partially known environment," Robot. Auton. Syst. 58(5), 425-434 (2010).

247. Z. Shiller, O. Gal and E. Rimon, "Safe Navigation in Dynamic Environments," In: Robot Design, Dynamics and Control (CISM Courses and Lectures) (W. Schiehlen and V. Parenti-Castelli, eds.) Vol. 524 (Springer, Vienna, 2010) pp. 225-232.

248. D. H. Shim, H. Chung and S. S. Sastry, "Conflict-free navigation in unknown urban environments," IEEE Robot. Autom. Mag. 13(3), 27-33 (2006).

249. D. H. Shim and S. Sastry, "An Evasive Maneuvering Algorithm for UAVs in See-and-Avoid Situations," Proceedings of the American Control Conference, Minneapolis, MN, USA (2007) pp. 3886-3891.

250. J. Shin and H. J. Kim, "Nonlinear model predictive formation flight," IEEE Trans. Syst. Man Cybern. Part A: Syst. Humans 39(5), 1116-1125 (2009).

251. A. M. Shkel and V. J. Lumelsky, "Incorporating body dynamics into sensor-based motion planning: The maximum turn strategy," IEEE Trans. Robot. Autom. 13(6), 873-880 (1997).

252. D. A. Shoenwald, "AUVs: In space, air, water, and on the ground," IEEE Control Syst. Mag. 20(6), 15-19 (2000).

253. R. Simmons, "The Curvature-Velocity Method for Local Obstacle Avoidance," IEEE International Conference on Robotics and Automation, Vol. 4, Minneapolis, MI, USA (Nov. 1996) pp. 33753382.

254. E. A. Sisbot, L. F. Marin-Urias, R. Alami and T. Simeon, "A human aware mobile robot motion planner," IEEE Trans. Robot. 23(5), 874-883 (2007).

255. E. Siva and J. M. Maciejowski, "Robust Multiplexed MPC for Distributed Multi-Agent Systems," Proceedings of the 18th IFAC World Congress, Milano, Italy (2011) pp. 251-256.

256. I. Skrjanc and G. Klancar, "Optimal cooperative collision avoidance between multiple robots based on Bernstein-Bezier curves," Robot. Auton. Syst. 58(1), 1-9 (2010).

257. J. Snape, J. van den Berg, S. J. Guy and D. Manocha, "Independent Navigation of Multiple Mobile Robots with Hybrid Reciprocal Velocity Obstacles," Proceedings of the IEEE/RSJ International Conference on Intelligent Robots and Systems, St. Louis, MO, USA (2009) pp. 5917-5922.

258. J. Snape, J. van den Berg, S. J. Guy and D. Manocha, "The hybrid reciprocal velocity obstacle," IEEE Trans. Robot. 27(4), 696-706 (2011).

259. R. Solea and U. Nunes, "Trajectory planning and sliding-mode control based trajectory-tracking for cybercars," Integr. Comput.-Aided Eng. 14(1), 33-47 (2007).

260. M. V. Srinivasan, S. W. Zhang, J. S. Chahl, E. Barth and S. Venkatesh, "How honeybees make grazing landings on flat surfaces," Biol. Cybern. 83(3), 171-183 (2000).

261. S. Srinivasan, K. Ramamritham and P. Kulkarni, "ACE, in the Hole: Adaptive Contour Estimation Using Collaborating Mobile Sensors," Proceedings of the International Conference on Information Processing in Sensor Networks, St Louis, MO, USA (Apr. 2008) pp. 147-158. 
262. C. Stachniss and W. Burgard, "An Integrated Approach to Goal-Directed Obstacle Avoidance Under Dynamic Constraints for Dynamic Environments," Proceedings of the 2002 IEEE/RSJ International Conference on Intelligent Robots and Systems, Vol. 1, Lausanne, Switzerland (2002) pp. 508-513.

263. D. M. Stipanovic, P. F. Hokayem, M. W. Spong and D. D. Siljak, "Cooperative avoidance control for multiagent systems," J. Dyn. Syst. Meas. Control 129(5), 699-707 (2007).

264. T. H. Summers and J. Lygeros, "Distributed Model Predictive Consensus via the Alternating Direction Method of Multipliers," Proceedings of the Allerton Conference on Communication, Control, and Computing, Monticello, IL, USA (2012) pp. 79-84.

265. S. Suri, E. Vicari and P. Widmayer, "Simple robots with minimal sensing: From local visibility to global geometry," Int. J. Robot. Res. 27(9), 1055-1067 (2008).

266. S. Susca, F. Bullo and S. Martinez, "Monitoring environmental boundaries with a robotic sensor network," IEEE Trans. Control Syst. Technol. 16(2), 288-296 (2008).

267. A. Tahirovic and G. Magnani, "PB/MPC Navigation Planner," In: Passivity-Based Model Predictive Control for Mobile Vehicle Motion Planning (Springer, London, 2013) pp. 11-24.

268. H. G. Tanner and A. Boddu, "Multiagent navigation functions revisited," IEEE Trans. Robot. 28(6), 1346-1359 (2012).

269. T. Tarnopolskaya, N. Fulton and H. Maurer, "Synthesis of optimal bang-bang control for cooperative collision avoidance for aircraft (ships) with unequal linear speeds," J. Optim. Theory Appl. 155(1), 115144 (2012).

270. H. Teimoori and A. V. Savkin, "A biologically inspired method for robot navigation in a cluttered environment," Robotica 28(5), 637-648 (2010).

271. H. Teimoori and A. V. Savkin, "Equiangular navigation and guidance of a wheeled mobile robot based on range-only measurements," Robot. Auton. Syst. 58(2), 203-215 (2010).

272. S. Thrun, "Learning occupancy grid maps with forward sensor models," Auton. Robots 15(2), 111-127 (2003).

273. J. M. Toibero, F. Roberti and R. Carelli, "Stable contour-following control of wheeled mobile robots," Robotica 27(1), 1-12 (2009).

274. B. Tovar, R. Murrieta-Cid and S. M. LaValle, "Distance-optimal navigation in an unknown environment without sensing distances," IEEE Trans. Robot. 23(3), 506-518 (2007).

275. W. Travis, A. T. Simmons and D. M. Bevly, "Corridor Navigation with a LiDAR/INS Kalman Filter Solution," Proceedings of the IEEE Intelligent Vehicles Symposium, Tokyo, Japan (2005) pp. 343-348.

276. C. Trevai, J. Ota and T. Arai, "Multiple mobile robot surveillance in unknown environments," Adv. Robot. 21(7), 729-749 (2007).

277. V. A. Tucker, "The deep fovea, sideways vision and spiral flight paths in raptors," J. Exp. Biol. 203(24), 3745-3754 (2001).

278. I. Ulrich and J. Borenstein, "VFH*: Local Obstacle Avoidance with Look-ahead Verification," Proceedings of the IEEE International Conference on Robotics and Automation, Vol. 3, San Francisco, CA, USA (2000) pp. 2505-2511.

279. USDoD, “Unmanned aircraft systems roadmap, 2005-2030," Technical Report, Office of the Secretary of Defense, Washington (2005).

280. V. I. Utkin, Sliding Modes in Control Optimization (Springer-Verlag, Berlin, 1992).

281. M. Vaccarini and S. Longhi, "Formation Control of Marine Veihicles via Real-time Networked Decentralized MPC," Proceedings of the 17th Mediterranean Conference on Control and Automation, Thessaloniki, Greece (2009) pp. 428-433.

282. L. Valbuena and H. Tanner, "Hybrid potential field based control of differential drive mobile robots," J. Intell. Robot. Syst. 68(3-4), 307-322 (2012).

283. J. van den Berg, S. J Guy, M. Lin and D. Manocha, Reciprocal n-Body Collision Avoidance, Springer Tracts in Advanced Robotics Series, Vol. 70 (C. Pradalier et al., eds.) (Springer, Berlin, Germay, 2011) pp. 3-19.

284. J. van den Berg and M. Overmars, "Planning time-minimal safe paths amidst unpredictably moving obstacles," Int. J. Robot. Res. 27(11-12), 1274-1294 (2008).

285. J. van den Berg, J. Snape, S. J. Guy and D. Manocha, "Reciprocal Collision Avoidance with AccelerationVelocity Obstacles," Proceedings of the IEEE International Conference on Robotics and Automation, Shanghai, China (2011) pp. 3475-3482.

286. J. van den Berg, D. Wilkie, S. J. Guy, M. Niethammer and D. Manocha, "Lqg-obstacles: Feedback Control with Collision Avoidance for Mobile Robots with Motion and Sensing Uncertainty," Proceedings of the IEEE International Conference on Robotics and Automation, St. Paul, MN, USA (2012) pp. 346-353.

287. A. C. Victorino, P. Rives and J.-J. Borrelly, "Safe navigation for indoor mobile robots. Part I: A sensor-based navigation framework," Int. J. Robot. Res. 22(12), 1005-1118 (2003).

288. M. P. Vitus, V. Pradeep, G. M. Hoffmann, S. L. Waslander and C. J. Tomlin, "Tunnel-MILP: Path Planning with Sequential Convex Polytopes," Proceedings of the AIAA Guidance, Navigation, and Control Conference, Minneapolis, MN, USA (2008) pp. 1-13.

289. N. A. Vlassis, N. M. Sgouros, G. Efthivolidis and G. Papakonstantinou, "Global Path Planning for Autonomous Qualitative Navigation," Proceedings of the IEEE Conference on Tools with Artificial Intelligence, Toulouse, France (Nov. 1996) pp. 354-359.

290. A. V. Savkin and H. Teimoori, "Decentralized navigation of groups of wheeled mobile robots with limited communication," IEEE Trans. Robot. 26(10), 1099-1104 (2010). 
291. Y. Wakasa, M. Arakawa, K. Tanaka and T. Akashi, "Decentralized Model Predictive Control via Dual Decomposition," Proceedings of the 47th IEEE Conference on Decision and Control, Cancun, Mexico (2008) pp. 381-386.

292. C. Wang, A. S. Matveev, A. V. Savkin, R. Cloutz and H.T. Nguyen, "A Real-time Obstacle Avoidance Strategy for Safe Autonomous Navigation of Intelligent Hospital Beds in Dynamic Uncertain Environments," Proceedings of Australasian Conference on Robotics and Automation (Dec. 2013).

293. C. Wang, A. S. Matveev, A. V. Savkin, T. N. Nguyen and H. T. Nguyen, "A Collision Avoidance Strategy for Safe Autonomous Navigation of an Intelligent Electric-Powered Wheelchair in Dynamic Uncertain Environments with Moving Obstacles," Proceedings of the European Control Conference, Zurich, Switzerland (Jul. 2013) pp. 4382-4387.

294. C. Wang, A. V. Savkin, T. N. Nguyen and H. T. Nguyen, "A Novel Algorithm for Safe Navigation of Intelligent Robotic Wheelchairs for Severely Disabled People in Crowded Dynamic Environments," Proceedings of the International Conference on Control, Automation, Robotics and Vision, Guangzhou, China (2012).

295. Y. Wang and G. S. Chirikjian, "A New Potential Field Method for Robot Path Planning," Proceedings of the IEEE International Conference on Robotics and Automation, Vol. 2, San Francisco, CA, USA (2000) pp. 977-982.

296. Z. Weihua and T. H. Go, "Robust decentralized formation flight control," Int. J. Aerospace Eng. available at: www.hindawi.com/journals/ijae/2011/157590/ (2011), online.

297. A. Widyotriatmo and K. Hong, "Navigation function-based control of multiple wheeled vehicles," IEEE Trans. Ind. Electron. 58(5), 1896-1906 (2011).

298. A. Wu and J. How, "Guaranteed infinite horizon avoidance of unpredictable, dynamically constrained obstacles," Auton. Robots 32(3), 227-242 (2012).

299. J. M. Yang and J. H. Kim, "Sliding mode control for trajectory tracking of nonholonomic wheeled mobile robots," IEEE Trans. Robot. Autom. 15(3), 578-587 (1999).

300. K. Yang, S. Gan and S. Sukkarieh, "An efficient path planning and control algorithm for RUAV's in unknown and cluttered environments," J. Intell. Robot. Syst. 57(1), 101-122 (2010).

301. X. Yang, L. Alvarez and T. Bruggemann, "A 3D collision avoidance strategy for UAVs in a non-cooperative environment," J. Intell. Robot. Syst. 70(1-4), 315-327 (2012).

302. T. Yata, L. Kleeman and S. Yuta, "Wall Following Using Angle Information Measured by a Single Ultrasonic Transducer," Proceedings of the IEEE International Conference on Robotics and Automation, Vol. 2, Leuven, Belgium (1998) pp. 1590-1596.

303. Y. Yoon, J. Shin, H. J. Kim, Y. Park and S. Sastry, "Model-predictive active steering and obstacle avoidance for autonomous ground vehicles," Control Eng. Pract. 17(7), 741-750 (2009).

304. H. Yu, R. Sharma, R. W. Beard and C. N. Taylor, "Observability-Based Local Path Planning and Collision Avoidance for Micro Air Vehicles using Bearing-only Measurements," Proceedings of the American Control Conference, San Francisco, CA, USA (2011) pp. 4649-4654.

305. Ch. Zhang, D. Arnold, N. Ghods, A. Siranosian and M. Krstic, "Source seeking with non-holonomic unicycle without position measurement and with tuning of forward velocity," Syst. Control Lett. 56(3), 245-252 (2007).

306. F. Zhang, D. M. Fratantoni, D. A. Paley, J. M. Lund and N. E. Leonard, "Control of coordinated patterns for ocean sampling," Int. J. Control 80(7), 1186-1199 (2007).

307. F. Zhang, E. W. Justh and P. S. Krishnaprasad, "Boundary Following Using Gyroscopic Control," Proceedings of the 43rd IEEE Conference on Decision and Control, Vol. 5, Paradise Island, Bahamas (2004) pp. 5204-5209.

308. F. Zhang and N. E. Leonard, "Cooperative control and filtering for cooperative exploration," IEEE Trans. Autom. Control 55(3), 650-663 (2010).

309. C. Zheng, L. Li, F. Xu, F. Sun and M. Ding, "Evolutionary route planner for unmanned air vehicles," IEEE Trans. Robot. 21(4), 609-620 (2005).

310. J. Zhipu and A. L. Bertozzi, "Environmental Boundary Tracking and Estimation Using Multiple Autonomous Vehicles," Proceedings of the 46th IEEE Conference on Decision and Control, New Orleans, LU, USA (Dec. 2007) pp. 4918-4923.

311. Y. Zhu and U. Ozguner, "Constrained Model Predictive Control for Nonholonomic Vehicle Regulation Problem," Proceedings of the 17th IFAC World Congress, Seoul, South Korea (2008) pp. 9552-9557.

312. Y. Zhu, T. Zhang and J. Song, "An Improved Wall Following Method for Escaping from Local Minimum in Artificial Potential Field Based Path Planning," Proceedings of the 48th IEEE Conference on Decision and Control and the 28th Chinese Control Conference, Shanghai, China (2009) pp. 6017-6022.

313. Y. Zhu, T. Zhang, J. Song and X. Li, "A new hybrid navigation algorithm for mobile robots in environments with incomplete knowledge," Knowl.-Based Syst. 27, 302-313 (2012).

314. B. D. Ziebart, N. Ratliff, G. Gallagher, C. Mertz, K. Peterson, J. A. Bagnell, M. Hebert, A. K. Dey and S. Srinivasa, "Planning-Based Prediction for Pedestrians," Proceedings of the IEEE/RSJ International Conference on Intelligent Robots and Systems, St Louis, MO, USA (2009) pp. 3931-3936. 